Gewichtsanalytische Bestinmung der Phosphorsäure als Iagnesimmpyrophosphat.

\title{
Yon \\ Z. Karaoglanow.
}

I. Allgemeiner Teil.

Zur Bestimmung. der Phosphorsäure als Magnesiumpyrophosphat sind mehrere Methoden vorgeschlagen worden. Die meisten von diesen Methoden beruhen auf Vereinbarungen, die anderen aber werden allgemein. angewandt. Wir werden hier nur die letzteren betrachter

Die alten Methoten von Abesser, Jani und Märker ${ }^{1}$, Peitsch, Rohn und Wagner2), Neubauer $\left.{ }^{3}\right)$, Gooch, und:

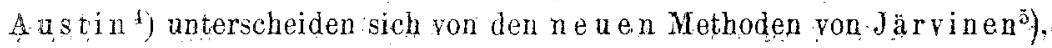
Jörgensen ${ }^{6}$ ) and Schmitzi hauptsächlich, dadurch, dass bei den alten Methoden die Fallung bei gewöhnlicher Temperatur, bei den neuen, unter Erhitzen yorgenommen wird. Bei fast allen Methoden zur Fallung; der Phosphorsäre als Magnesiumammoniumphosphat wird die Fallung: langsam und unter beständigem Umrühren der Fällungslösung ausgeführt.

Yie yerschiedenèn Methoden zur Fällung dèr Phosphơrsäure als Magnesiumammoniumpliosphat unterscheiden sich tnteveinander durch die: Zusammensetzung der zu. fällendèn Lösung; and des Fällungsmittels. Die "Hauptbestandteile dieser Lösungen sind Phospharsäure, Magnesiụmchlorid, Ammoniumchlorid und Ammoniak. Hierans werden die Fiallungslossung und das Fälungsmittel bei den verschiedenen Methoden anf verschiedene Weise hergestellt.

1) Diesé Ztschrft. 12, 239 (1873).

) Diese Ztschrft. 19, $444(1880)$.

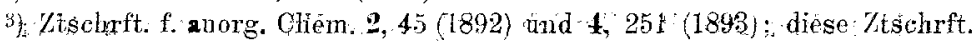
3ื3, 362 (1894).

4) Ztschrft. f. anorg. Chem 20, 121 (1891

5) Diese Ztschrft. 14, 3333 (1905).

6) Diese Ztsehrft. 45, 273 (1906); 46, 370 (1907); Ztschrft.w. f. angew. Chem. 24, s42 (1911): diese Ztgchrft. 50,336 (1911)

i) Diese Ztschrft. 15, 512 (1901i):

Fresonis, Zeitschrift $\mathrm{f}$ anal. Miemie. TATI. Jahrgang. 11, u, 12. Heft. 3 
Es fragt sich nun: Von welcher Bedeutung die Reihenfolge ist, nach welcher die sicb an dem Fällungsvorgang beteiligenden Stoffe miteinander gemischt werden? Welches ist die günstigste Temperatur, bei welcher die Fällung ausgeführt werden muss? Warum muss die Fällung der Phosphorsänre tropfenweise geschehen?

Die langsame Fállung entspricht unzweifelhaft nicht der üblichen Arbeitsweise in der analytischen Praxis. Sie wird in derselben nur dann vorgenommen, wenn dadurch sekundäre Fällungsvorgänge vermieden werden sollen. Es folgt tatsächlich aus den Untersuchungen von Neubauer (1. c.) einerseits und Gooch und A ustin (1. e.) andererseits, dass beim Fällen der Phosphorsäure als Màgnesiumammoniumphosphat sekundäre Fällungsvorgänge verlaufen. Es entsteht nun die Frage: Sind diese sekundären Vorgänge bei den neuen Methoden zur Bestimmung der Phosphorsäure als Magnesiumpyrophosphat vermieden und welche Bedingungen begünstigen ihre Entstehung? Die Untersuchungen von Neabauer, Gooch und A ustin und neverdings von K. Bubel) and D. Balarew ${ }^{2}$ ) haben zwar viel zur Aufklärung dieser theoretisch und praktisch wichtigen Fragen beigetragen, aber zu ihrer endgültigen Lösung haben sie nicht geführt.

Bevor wir auf die experimentelle Untersuchung dieser Fragen eingehen, wollen wir theoretisch die sekundären Vorgänge betrachten, welche beim Fällen der Phosphorsäure als Magnesiumammoniumphosphat möglich sind. Unter den Bedingungen, bei welchen die Phosphorsäure als Magnesiumammoniumphosphat gefällt wird, sind zwei Arten von sekundären Vorgängen möglich : 1. Fällung von Magnesiumbydrat und 2. Fällung von solchen sekundären Produkten, welche durch die Ionenzusammensetzung der Phosphorsänre und des Magnesiumchlorids bedingt siud. Wie aus dem Folgenden zu ersehen ist, sind bei den verschiedenen Methoden die Bedingungen für die Entstehung dieser sekundären Produkte nicht gleich. Da mit den Methoden von Järvinen, Jörgensen und Schmitz die drei wichtigsten Möglichkeiten erschöpft sind, nach welchen die Fällungslösung und das Fällungsmittel bereitet werden können, so werden wir nur diese drei theoretisch betrachten.

In meiner Arbeit *Gewichtsanalytische Untersuchungen über ein-, zwei- und drei-basische Säuren ${ }^{3}$ ), habe ich theoretisch die sekundären Vorgänge, welche beim Fällen von Phosphorsäure als Magnesium-

1) Diese Ztschrft. 49, 525 (1910).

2) Ztschrft. f. anorg. Chem. 97, 149 (1916) und 101, 229 (1917).

3) Jahrbuch der Universität in Sufia X und XI (1915). 
ammoniumphosphat denkbar sind, behandelt. Kurz zusammengefasst ergab sich, dass, wenn die Fällungslösung Ammoniumphosphat $\left(\mathrm{NH}_{4}\right)_{3} \mathrm{PO}_{4}$ enthält und das Fällungsmittel Magnesiumchlorid ist, beim Vermischen der beiden Lösungen ausser dem Hauptvorgang

$$
\mathrm{NH}_{4} \mathrm{PO}_{4}{ }^{\prime \prime}+\mathrm{Mg}{ }^{\circ}=\mathrm{Mg} \mathrm{NH} \mathrm{PO}_{4}
$$

noch folgende sekundäre Ionenvorgänge denkbar sind:

$$
\begin{aligned}
\left(\mathrm{NH}_{4}\right)_{2} \mathrm{PO}_{4}{ }^{\prime}+\mathrm{Mg} \mathrm{Cl} & =\mathrm{MgCl}\left(\mathrm{NH}_{4}\right)_{2} \mathrm{PO}_{4} \\
2\left(\mathrm{NH}_{4}\right)_{2} \mathrm{PO}_{4}{ }^{\prime}+\mathrm{Mg}{ }^{\circ} & =\mathrm{Mg}\left[\left(\mathrm{NH}_{4}\right)_{2} \mathrm{PO}_{4}\right]_{2} \\
\mathrm{NH}_{4} \mathrm{PO}_{4}{ }^{\prime \prime}+2 \mathrm{Mg} \mathrm{Cl} & =(\mathrm{Mg} \mathrm{Cl})_{2} \mathrm{NH}_{4} \mathrm{PO}_{4} \\
\mathrm{PO}_{4}{ }^{\prime \prime}+3 \mathrm{Mg} \mathrm{Cl} & =(\mathrm{Mg} \mathrm{Cl})_{3} \mathrm{PO}_{4} \\
2 \mathrm{PO}_{4}{ }^{\prime \prime \prime}+3 \mathrm{Mg} & =\mathrm{Mg}_{3}\left(\mathrm{PO}_{4}\right)_{2} .
\end{aligned}
$$

Von diesen theoretisch denkbaren Produkten kann die Verbindung $\mathrm{MgCl}\left(\mathrm{NH}_{4}\right)_{2} \mathrm{PO}_{4}$ als ein Gemisch von Magnesiumammoniumphosphat und Ammoniumchlorid betrachtet werden, infolgedessen wird ihre Gegenwart im Niederschlag keinen Einfluss auf die analytischen Resultate ausüben. Die Verbindung $\mathrm{Mg}\left[\left(\mathrm{NH}_{4}\right)_{2} \mathrm{PO}_{4}\right]_{2}$ wird bei hoher Temperatur nach der Gleichung

$$
\mathrm{Mg}\left[\left(\mathrm{NH}_{4}\right)_{2} \mathrm{PO}_{4}\right]_{2}=\mathrm{Mg}\left(\mathrm{PO}_{3}\right)_{2}+4 \mathrm{NH}_{3}+2 \mathrm{H}_{2} \mathrm{O}
$$

zerfallen, folglich wird ihre Gegenwart im Niederschlag niedrigere analytische Resultate zar Folge haben. Die Verbindung $(\mathrm{Mg} \mathrm{Cl})_{\mathbf{2}} \mathrm{NH}_{4} \mathrm{PO}_{4}$ wird wahrscheinlich nach der Gleichung

$$
2(\mathrm{Mg} \mathrm{Cl})_{2} \mathrm{NH}_{4} \mathrm{PO}_{4}=(\mathrm{Mg} \mathrm{Cl})_{4} \mathrm{P}_{2} \mathrm{O}_{7}+2 \mathrm{NH}_{3}+\mathrm{H}_{2} \mathrm{O}
$$

zerfallen und wird höhere analytische Resultate hervorrufen. Zuletzt

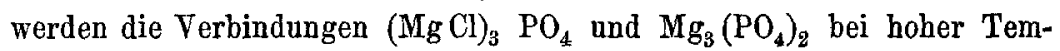
peratur unverändert bleiben und gleichfalls $z \mathbf{u}$ höheren analytischen Resultaten führen.

Die Bedingungen, unter welchen die Fällung der Phosphorsäure als Magnesiumammoniumphosphat nach den erwähnten Methoden erfolgt, sind noch verwickelter. Z. B. wird nach der Methode von Järvinen die ammoniakalische Phosphatlösung mit einer Lösung gefällt, welche gleichzeitig Magnesiumchlorid, Ammoniumchlorid und Ammoniak enthält. Nach der Methode von Jörgensen enthält die zu fällende Lösung Phosphorsäure, Ammoniak und Ammoniumchlorid und das Fällungsmittel - Magnesiumchlorid. Es kann trotzdem angenommen werden, dass der oben angegebene Verlauf des Fällungsvorgangs für die Methoden von Järvinen und Jörgensen und für die diesen ähnlichen gilt. 
Die besonderen Umstände, unter weỉchen der Fällungsvorgang bei der Methode vou Sehmitz vor sich geht, bedingen einen anderen Vertauf der chemischen Umsetzungen. In diesem Falle enthält die zu fällende Lösung Phosphorsäure, Ammoniumchlorid, Magnesiumchlorid und Salzsiure und das Fällungsmittel ist Ammoniak. Wird Ammoniak zu der Fällungslösung zugesetzt, so wird erst die überschüssige Salzsäure neutralisiert und es entstehen günstige Bedingungen für die gegenseitige Einwirkung von freier Phosphorsẳure und Magnesiumchlorid. Da die Phosphorsäure ein 'schwacher Elektrolyt ist, "so wird ihre Lösung eine grössere Konzentration von den Anionen $\mathrm{H}_{2} \mathrm{PO}_{4}^{\prime}$ und $\mathrm{HPO}_{4}$ " enthalten. In diesem Falle wird der Hauptvorgang der folgende sein:

$$
{ }^{\mathrm{H}} \mathrm{PO}_{4} " \mathrm{Mg}+\mathrm{Mg} \mathrm{H} \mathrm{PO}_{4}
$$

und die sekundären $Y$ organge werden sich zwischen den Kationen $\mathrm{Mg}$. und $\mathrm{MgCl}$ and den "thionen $\mathrm{H}_{2} \mathrm{PO}_{4}$ " H $\mathrm{PO}_{4}^{\prime \prime}$ und $\mathrm{PO}_{4}^{\prime \prime}$ abspielen (vergl., S. 499).

In wie fern der Hauptrorgang von sekundären Vorgängen begleitet sein wird, wird von der Lôslichkeit der sekundüren Produkte abhängen. Trotzdem die Eigenschaften der hypothetischen sekundären Produkte nïcht bekannt sind; kann "man im Voraus sagen," dass die sauren Magnesiumphosphate leichter löslich sein werden, als die entsprechenden Ammoniummağnesiumphosphate. "Andererseits sind die Bedingungen für die Entstehung der Produkte $\mathrm{Mg}_{3}\left(\mathrm{PO}_{4}\right)_{2}$ und $(\mathrm{Mg} \mathrm{Cl})_{3} \mathrm{PO}_{4}$ bei der Fällung nach der Methode yon Schmitz ungünstig, weil hierbel der grössite Teil des Fällurgsvorganges in saurer Lösung verlänft.

Nachdem man zar Fällungslösung einen kleinen Übersehuss von verdüntem Ammoniak zugesetzt hat, lässt man, nach der Methode yon. Schmitz, die Flussigkeit erkalten, und damn wird ein grosser Uerschuss yon konzentriertem Ammoniak zugefügt. Es ist. klar; dass bei diesen Bedingungen die sauren Phosphate in die entsprechenden-Ammoniummagnesiumphosphate übergehen werden, Dies wird natürlich in dem Malse- stattinden, als der Niederschlag solche sanren Phosphate enthält. Es folgt aus dem Gesagten, dass beim Fallen dez Phosphorsäure nach dep Methoderon Schmitz manehe setkudären Yorgänge beseitigt oder wenigstens begrenzt werden, it a deren Eintritt die Bedingungeg bei den Methoden yonjarvinen und Jörgensen güntiger sinde.

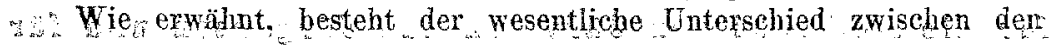
alten und den neven Methoden zur Fällung der Phosphorsäure als 
Magnesiumammoniumphosphat darin, dass bei den ersteren kalt und bei den letzteren heiss gefällt wird. Unter der Voraussetzung, dass die Löslichkeit : der denkbaren sekundären Fällungsprodakte mit höherer Temperatur zunimmt, kommen wir zu dem Sehluss, dass bei hoher Temperatur der Übergang der sekundären Produkte in das am schwersten lösliche Magnesiumammoniumphosphat erleichtert wird. Mit anderen Worten: Bei der heissen Fällung sind die Bedingungen zur Entstehung yon sekundären Produkten ungünstiger, ganz abgesehen davon, nach welcher Methode die Fällung a usgeführt wird.

Was diè Entstehung von Magnesiumhydrat als selkundäres Produkt anbelangt, so sind die Bedingungen hierfür bei den versehiedenen Methoden zur Fällung der Phosphorsäure gleichfalls nicht dieselben, Das wird aus folgendem klar:

Nach der Methode yon Järvinen wird zu derammoniakalischen Lösung des Phosphats tropfen weise eine Lösung zugesetzt, welche A mmoniumchlorid, Magnesiumchlorid und Ammoniak enthält.' Dass hierbei auch Magnesiumhydrat gefällt werden kann, ergibt sich aus Folgendem: Beim Zusatz des Fällungsmittels zu der Fällungslösung nimmt die Konzentration des Ammoniumchlorids stark ab, während die Konzentration des Ammoniaks verbältnismälsig nicht so stark abnimmt. Dadurch entstehen günstige Bedingungen zur Fällung von Magnesiumbydrat. Das Löslichkeitsprodukt des Magnesiumammoniumphosphats ist kleiner als dasjenige des Magnesiumhydrats. Andererseits ist die Löslichkeit des Magnesiumhydrats bedeutend grösser bei Gegenwart von Ammoniumchlorid. Man könnte also erwarten, dass sich das im Anfang gebildete Magnesiumbydrat später lösen würde: Da aber die heterogenen Vorgänge nicht so glatt verlaufen, ist es nicht angeschlossen, dass in den Niederschlag ron Magnesiumammoniumphosphat Magnesiumhydrat über. geht.

Bei der Methode y on Jörgensen sind die Bedingungen făr die Entstebung von Magnesiumbydrat weniger günstig, da die Fällungslösung von Anfang an Ammoniumchlorid enthält. Noch ungünstiger sind die Bedingungen zur Bildung von Magnesiumbydrat als sekundäres Produkt bei der Methode von Schmitz und zwar nicht bloss deswegen, weil die Fällungslösung Ammoniumchlorid enthält, sondern auch deswegen; weil der Fällungsvórgañg hauptsächlich in sauerer Lösung verläuft. 


\section{Einfluss der Fällungsdauer.}

Angenommen, dass die Fällungsdauer beim Fällen der Phosphorsäure nach der Methode von Järvinen von Einfluss ist und dass dabei nur solche sekundären Vorgänge möglich sind, welche durch die Ionen des Phosphats und des Magnesiumchlorids hervorgerufen werden, so muss man erwarten, dass, wenn die Fällung rasch ausgeführt wird, solche sekundären Vorgänge verlaufen, an welchen die zusammengesetzten Ionen $\mathrm{MgCl}^{\circ}$ und $\mathrm{H}_{2} \mathrm{PO}_{4}{ }^{\prime}$ beteiligt sind. Wird die Fällung nach der

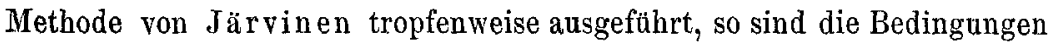
für die Beteiligung der Ionen $\mathrm{Mg} \mathrm{Cl}$ und $\left(\mathrm{NH}_{4}\right)_{2} \mathrm{PO}_{4}{ }^{\prime}$ an dem Fällungsvorgang ungünstiger, weil dadurch dem Phosphat und dem Magnesiumchlorid die Möglichkeit gegeben wird, sich vollständiger zu ionisieren. Beim langsamen Fällen entstehen aber günstigere Bedingungen für das Eintreten von solchen sekundären Vorgängen, welche durch das Anion $\mathrm{PO}_{4}{ }^{\prime \prime \prime}$ hervorgerufen werden.

Was die Entstehung von Magnesiumhydrat als sekundäres Produkt anbelangt, so sind (nach dem oben gesagten) bei der Methode von Järvinen die Bedingungen für seine Entstehung günstiger, wenn die Fällung langsam ausgeführt wird.

Die Ionenzusammensetzung der Lösungen bei der Methode von Jörgensen ist im grossen und ganzen dieselbe, wie bei der Methode von Järvinen. Folglich werden auch die sekundären Vorgänge, welche durch die Fällungsdauer hervorgerufen werden können, bei den beiden Methoden qualitativ gleich sein. Eine eingehendere Betrachtung der Zusammensetzung der Lösungen bei den beiden Methoden zeigt aber, dass in quantitativer Beziehung die Ionen nicht gleich sein werden. Bei der Methode von Jörgensen enthält die zu fällende Lösung nicht bloss mehr Ammoniak; sondern auch noch Ammoniumchlorid. Infolgedessen wird die Konzentration der Anionen $\left(\mathrm{NH}_{4}\right)_{2} \mathrm{PO}_{4}{ }^{\prime}$ and $\mathrm{NH}_{4} \mathrm{PO}_{4}{ }^{\prime \prime}$ grösser und die Konzentration des Anions $\mathrm{PO}_{4}{ }^{\prime \prime}$ kleiner sein, als bei der Methode von $J$ ärvinen. Es ist klar, dass auch die quantitative Ionenzusammensetzung des Fällungsmittels bei den Methoden von Järvinen und $J$ örgensen nicht gleich sein kann.

Wird dieser Unterschied in der Ionenzusammensetzung der Lösungen bei beiden Methoden berücksichtigt, so kommt man zu dem Schluss, dass beim langsamen Fällen nach der Methode von Jörgensen die Bedingungen fär die Entstehung von $\mathrm{Mg}_{3}\left(\mathrm{PO}_{4}\right)_{2}$ un-- 
günstiger und beim raschen Fällen die Bedingungen für die Bildung von $\mathrm{Mg}\left[\left(\mathrm{NH}_{4}\right)_{2} \mathrm{PO}_{4}\right]_{2}$ günstiger sind, als bei der Methode von Järvinen. Für die Fällung von Magnesiumhydrat als sekundäres Produkt sind bei der Methode von Jörgensen die Bedingungen ungünstig, gleichgültig ob rasch oder langsam gefällt wird.

Wenn die Fällung nach der Methode ron Schmitz rasch ausgeführt wird, so kann man solche sekundären Vorgänge erwarten, wie sie von dem Kation $\mathrm{Mg} \mathrm{Cl}^{*}$ hervorgerufen werden. Beim langsamen Fällen nach derselben Methode sind, wie wir gesehen haben, die Bedingungen für den Verlauf von sekundären Vorgängen nicht günstig. Das bisher über den Einfluss der Fällungsdauer Gesagte, gilt nur für den Fall, dass die sekundären Vorgänge gleichzeitig von den Ionen der Phosphorsäure und des Magnesiumchlorids veranlasst werden. Angenommen, dass das Kation $\mathrm{MgCl}^{*}$ sich nicht an den Fällungsvorgängen beteiligt, und dass beim Fällen der Phosphorsäure als Magnesiumammoniumphosphat die Entstehung von Magnesiumbydrat ausgeschlossen ist, so kommt man zu anderen Schlüssen in bezug auf den Einfluss der Fällungsdauer.

In diesem Falle werden nur die Ionen der Phosphorsäure oder des Phosphats sekundäre Vorgänge hervorrufen kënnen. Dann ist theoretisch zu erwarten, dass beim raschen Fällen diejenigen sekundären Vorgänge verlaufen werden, welche durch die zusammengesetzten Ionen der Phosphorsäure bedingt sind. Meine bisherigen Untersuchungen ${ }^{1}$ ) über den Einfluss der Fällungsdauer haben aber gezeigt: 1. dass die langsame Fällung nur dann unentbehrlich für die analytische Praxis ist, wenn dadurch solche sekundären Vorgänge vermieden werden müssen, die durch die Ionenzusammensetzung des Fällungsmittels veranlasst sind, und 2. dass, wenn die sekundären Vorgänge nur durch die Ionenzusammensetzung der zu fällenden Lösung hervorgerufen werden, die Fällungsdauer keine grosse Rolle bei dem Verlauf der Fällungsvorgänge spielt. Von diesen experimentellen Grundsätzen und von den obigen Annahmen ausgehend, kommt man zu dem Schluss, dass der Unterschied zwischen den analytischen Resultaten, welche einerseits beim raschen und andererseits beim langsamen Fällen der Phosphorsäure erhalten werden, nicht gross sein kann, und dass sogar die Resultate genauer ausfallen werden, wenn die Fällung raseh ausgeführt wird.

1) Diese Ztsehrft. 57, 77, 113 (1918). 


\section{Experimenteller Teil.}

1. Es folgt aus dem oben Gesagten, dass beim Fälen der Phosphorsäare als Magnesiumammoniumphosphat sekundäre Fällungsvorgänge denkbar sind, welche positive oder negative analytische Fehler hervorrufen können. $O b$ in der Tat solche Vorgänge verlaufen, kann nicht im Voraus gesagt werden. Die Bedingungen, unter welchen die Fäliung der Phosphorsäure als Magnesiumammoniumphosphat nach den verschiedenen Methoden geschieht, sind verwickelt, infolgedessen wäre es schwierjg, auch wenn die Figenschaften der möglichen sekundären Produkte beKannt wären, vorauszuseben, wie sich dieselben unter dem Einfluss diesor Bedingungen verändern würden. Die sicherste Kontrolle der theoretischen Folgerungen ist nur experimentell möglich und zwar dadurch, dass man vergleichende analytische Versuche nach den verschiedenen Methoden ausführt. Bei diesen Untersuchungen habe ich als Ausgangslösung eine Lösung von freier und reiner Phosphorsäure angewandt. Von dieser Lobsung wurden für jeden Yersuch genau $50 \mathrm{~cm}$ abgemessen. Es wurden ausserdem noch folgende Lösungen benutzt:

Magnesiumchlorid, enthaltend $55 \mathrm{~g} \mathrm{MgCl} .6 \mathrm{H}_{2} \mathrm{O}$ im Liter. Die Lösung ist schwach mit Salzsäure angesäuert.

Ammoniumchlorid, gesättigt bei gewöhnlicher Temperatur.

Ammoniak, $2,5 \%$ ige Lösung.

Ammoniak, konzentrierte Lösung.

Ammoniummolybdat, enthaltend $120 \mathrm{~g}\left(\mathrm{NH}_{4}\right)_{6} \mathrm{Mo}_{7} \mathrm{O}_{24} 4 \mathrm{H}_{2} \mathrm{O}$ in einem ?. Ein $c c m$ vón dieser Lösung fällt ungefähr $0,004 g \mathrm{P}_{2} \mathrm{O}_{5}$ aus. Kaliumchloria, gesättigt bei gewöhnlicher Temperatur.

Natriumchlorid, gesättigt bei gewöhnlieher Temperatur.

Salzsäure $6 \mathrm{n}$.

Alle Lösungen wurden aus reinen Präparaten hergèstelit.

Aus diesen Lösungen wurden die Fällungslösung und das Fällungsmittel bereitet und zwar auf verschiedene Weise, je nach der Methode, "nach welcher die Fällung geschehen sollte. Die zu fällende Lösung wurde immer mit einer bestimmten Menge Wasser verdunnt. Nachdem dieselbe anf die gew ünschte Temperatur gebracht war, wurde gefällt. Das Făllungsmittel wurde îe erwärmt. Bei den raschen Fâllungen wurde das Fällungśmittel mỏglichst rasch zu der Fällungslösung gegossen. Bei den Iâng samen Fällungen wurde das Fällungsmittel aús einer Bürette oder aús einem Scheidetrichter in die Fällungslösung tropfen gelassent Wäăhend 
der Fällung wurde die Lösung des Phosphats stets mit dem Glasstab́ gerührt. Alle Versuche sind in Bechergläsern aus Jenaer Glas aus+ geführt:

Nach der Fällung wurde die Flüssigkeit mit dem Niederschlag erkalten gelassen und dann, wenn die Methode es verlangte, konzentriertes Ammoniak zugesetzt. Während des Erkaltens wurde der Niederschlag oft gerührt. Das Filtrieren erfolyte gewöhnlich etwa 4 oder 16 Stunden nach der Fällung. Da es sich gezeigt hat, dass dieser Umstand ohne Einfluss auf die Resultate ist, werde ich bei den einzelnen Yersuchen nicht angeben, wie viel Stunden nach der Fällung filtriert worden ist.

Sämtliche Niederschläge wurden durch gewöhnliche Filter filtriert. Was Waschen der Niederschläge erfolgte stets mit $2,5^{0}{ }_{0}$ igem Ammoniak und dauerte so lange; bis im Filtrat keine Chlorionen mehr nachgewiesen werden konnten.

Einige Eigenschaften des Magnesiumammonium phosphats. Das Aussehen des Magnesiumammoniumphosphats, welches durch Fällen von Phosphorsäure erhalten wird, ist verschieden und hängt davon $a b$, unter welchen Bedingungen die Fällung geschieht. Bei sehr langsamem Fällen erhält man den Niederschlag gleich im kristallinischen Zustand, gleichgültig, ob die Fällung bei hoher oder bei gewöhnlicher Temperatur geschieht, und unabhängig davon, nach welcher Methode gefällt wird. Die bei gewöhnlicher Temperatur erhaltenen Niederschläge zeichen sich dadurch aus, dass sie viel feinkristallinischer sind. Wenn die Fällung nicht sehr langsam geschieht, hängt das Aussehen des Magnesiumammoniumphosphats von der Zusammensetzung der Lösungen ab.- Unter gewöhnlichen Bedingungen in bezug auf die Konzentration des Magnesiumchlorids, Ammoniumchlorids und des Ammoniaks erhält man einen im Anfang amorphen Niederschlag, der aber schnell in den kristallinischen Zustand übergeht. Wenn die zu fällende Lösung grosse Mengen ron Ammoniuntehlorid oder Ammoniummolybdat enthält, so fängt die Niederschlagsbildung erst an, nachdem verhältnismälsig viel Fällungsmittel zugesetzt' worden ist, und der Niederschlag ist dann gleich kristallinisch. Wenn aber die Lossung viel Magnesiumchlorid enthält, so wird dadurch der Ubergang des àmorphen Niederschlags in den kristallinischen Zustand ersehwert.

Interessanter sind die Erscheinungen, welche beim raschen Fällen beobachtete werder. - In diesem Falle sind die Niederschläge im Anfärg fast imner amorph sie haben ein grosses Volumen und setzen sich 
langsam ab. Beim Stehen gehen die amorphen Niederschläge in den Kristallzustand über, wobei ihr Volumen stark abnimmt. Dieser Übergang verläuft schneller, wenn die Flüssigkeit grosse Mengen von Ammoniumchlorid oder Ammoniummolybdat enthält, und wird durch grosse Mengen von Magnesiumchlorid verlangsamt.

Wenn die zu fällende Lösung sehr grosse Mengen von Ammoniumchlorid oder Ammoniummolybdat enthält, so wird folgende Erscheinung beobachtet. Nach dem raschen Zusatz des Fällungsmittels bleibt die Lösung klar, trotzdem das Fällungsmittel im Überschuss vorhanden ist, und erst nach einigen Sekunden fängt die Bildung des Niederschlages an. Das unter solchen Bedingungen entstandene Magnesiumammoniumphosphat erscheint gleich im Kristallzustand. Diese Erscheinung wird häufiger, namentlich dann beobachtet, wenn die Fällung nach der Methode von Schmitz ausgeführt wird; sie findet seltener statt, wenn die Fällung nach der Methode von Jörgensen erfolgt, und wird nicht beobachtet beim Fällen nach Järvinen. Die Gegenwart von viel Magnesiumchlorid in der Fällungslösung verhindert ihre Entstehung. Auch in diesem Falle sind beim kalten Fällen die Niederschläge feiner kristallinisch.

Das Volumen des Niederschlags von Magnesiumammoniumphosphat ist verschieden, je nach den Bedingungen, unter welchen die Fällung erfolgt. Überhaupt haben die Niederschläge ein grösseres Volumen, wenn kalt gefällt wird, oder wenn die Lösung während der Fällung andauernd gekocht wird. Das Volumen der Niederschläge hängt weiter $a b$ : 1. von der Fällungsdauer. Jas Volumen ist grösser, wenn die Fällung langsam ausgeführt wird; 2. von der Methode, nach welcher gefällt wird. Die Niederschläge haben das grösste Volumen, wenn sie nach Järvinen und das kleinste, wenn sie nach Schmitz gefällt sind; 3. von der Zusammensetzung der Lösungen. Das Volumen der Niederschläge ist kleiner, wenn die zu fällende Lösung grössere Mengen von Ammoniumchlorid oder Ammoniummolybdat enthält, und es ist grösser, wenn sie viel Magnesiumchlorid enthält.

Die Eigenschaft des Magnesiumammoniumphosphats, an den Glaswänden zu haften, hängt gleichfalls von den Bedingungen $a b$, unter welchen es entsteht. Es zeigt diese Eigenschaft nicht, wenn die Fällung bei gewöhnlicher Temperatur erfolgt. Es bleibt aber um so mehr haften, je höher die Temperatur ist. Auch die Art der Fällung ist dabei von Bedeutung. Das Haften am Glase ist am stärksten bei solchem Magnesiumammoniumphosphat, welches nach der Methode von Schmitz erhalten wird. Wir werden sehen, dass diese Eigenschaften des Nieder- 
schlags von Magnesiumammoniumphosphat in Zusammenhang mit den analytischen Resultaten und folglich auch mit der Zusammensetzung des Niederschlages stehen.

Der ausgewaschene Niederschlag von Magnesiumammoniumphosphat wurde im Trockenschrank getrocknet, vom Filter getrennt, das Filter verascht und zu der Asche das Magnesiumammoniumphosphat zugefügt. Das Glühen erfolgte im Anfang vorsichtig mit gewöhnlichem Brenner und dauerte etwa $1 / 4$ Stunde. Dann wurde im elektrischen Ofen je $1 / 2$ Stunde bis zu konstantem Gewicht geglüht. Alle Versuche wurden in Platintiegel ausgeführt.

Beim Glühen nimmt das Volumen des Magnesiumammoniumphosphats in den meisten Fällen wesentlich ab, es wird erst gelblich, dann gerät es in Glut, und man erhält graues bis schwarzes Magnesiumpyrophosphat, welches hart ist, lavaähnliche Struktur hat und ein kleines Volumen besitzt. Solches Magnesiumpyrophosphat wird beim Glühen mit dem gewöhnlichen Brenner nicht heller, es wird aber weiss bei andauerndem und starkem Glühen im elektrischen Ofen oder auf dem Gebläse.

Magnesiumammoniumphosphat aber, welches unter bestimmten Bedingungen erhalten wird, zieht sich beim Glühen nicht so stark zusammen, es wird bei hoher Temperatur dunkel, aber dann noch beim Glühen mit dem gewöhnlichen Brenner wieder hell. Solches Magnesiumammoniumphosphat gerät beim Kalzinieren nicht in Glut und das aus demselben erhaltene Magnesiumpyrophosphat ist gewöhnlich ganz weiss, besitzt kristallinische Struktur und ein grosses Volumen.

Das gefürbte Magnesiumpyrophosphat wird beim starken Glühen, oder beim Verdampfen mit Salzsäure weiss, ohne dass dabei sein Gewicht merkliche Änderung erleidet. A uf Grund dieser Tatsache habe ich beim Wägen auf die Farbe des Glührückstandes nicht geachtet.

Sämtliche Versuche in dieser Arbeit sind reihenweise angestellt. Jede Reihe bestand aus 8 bis 10 Bestimmungen, bei welchen die Fällung, das Waschen des Niederschlages usw. unter vollständig gleichen Bedingungen ausgeführt wurde. Die einzelnen Versuche, welche eine Reihe bilden, unterscheiden sich nur in solchen Punkten, deren Einfluss auf den Verlauf des Fällungsvọgangs geprüft werden sollte.

Fällung der Phosphorsäure als Magnesiumammoniumphosphat bei hoher Temperatur.

Aus der Literatur, die mir zur Verfügung steht, ist nicht ersichtlich, aus welchen Gründen bei den alten Methoden die Fällung der Phosphor- 
săure- bei gewöhnlicher Temperatur und bei den neuen Methoden bei hoher Temperatur ausgeführt wird. "Auch aus den neueren Arbeiten Järvinens, Jörgensens und Schmitz ist nicht $z$ a ersehen, welches die günstigste Temperatur ist, bei welcher die Fällung geschehen soll, Bei einer Reihe von Versuchen, welche nach den genannten Methoden ausgeführt wurden, habe ich bemerkt, dass unter sonst gleichep Versuchsbedingungen die Resultate davon abhängig sind, bei welcher Temperatur die Fällung geschieht. Zur Erforschung des Temperatur einflusses auf die analytischen Resultate wurde ich hauptsächlich dadurcla veranlasst, weil das unter bestimmten Temperaturbedingungen erhaltene Magnesiumammoniumphosphat beim Übergehen in:Magnesiumpyrophosphat nicht in Glut gerät. Über den Zusammenhang zwischen der Eigenschaft des Magnesiumammoniumphosphats, beim Kalzinieren za erglühen und seiner Zusammensetzung ist in einer anderen Arbeit ${ }^{1}$ ) berichtet worden.

Erste Versuchsreihe.

\section{A. Fällung der Phosphorsäure ohne Erwärmen während des Fällens:}

Bei dieser Versuehsreihe wurden die Fällungslösung und das Fällungsmittel nach der entsprechenden Methode bereitet, dann die zu făllende Lösung bis zum Kochen erhitzt und, ohne weiter zu erwärmen, gefält.

1. Versuche nach der Methode von Järvinen.

Tabelle 1.

Einfluss der Konzentration und der Fälungsdauer.

\begin{tabular}{|c|c|c|c|c|c|c|c|c|}
\hline \multirow{2}{*}{$\mathrm{Nr}$} & $F \ddot{a}$ & ngslö & & & llungsmi & & \multirow{2}{*}{$\begin{array}{l}\text { Fällungs- } \\
\text { dauer }\end{array}$} & \multirow{2}{*}{$\begin{array}{c}\text { Ge- } \\
\text { funden } \\
\mathrm{Mg}_{2} \mathrm{P}_{2} \mathrm{O}_{7} \\
g\end{array}$} \\
\hline & $\begin{array}{c}\mathrm{H}_{3} \mathrm{PO}_{4} \\
\text { ecm }\end{array}$ & $\begin{array}{r}\mathrm{H}_{2} \mathrm{O} \\
\mathrm{cm} \\
\end{array}$ & $\begin{array}{l}\mathrm{NH}_{3} \\
c c m \\
\end{array}$ & $\begin{array}{c}\mathrm{MgCl}_{2} \\
\mathrm{ccm} \\
\end{array}$ & $\begin{array}{c}\mathrm{NH}_{4} \mathrm{Cl} \\
\mathrm{ccm}\end{array}$ & $\begin{array}{l}\mathrm{NH}_{3} \\
\mathrm{ccm}\end{array}$ & & \\
\hline 1 & 50 & 50 & 5 & 50 & 30. & 15 & 15 Min. & 0,4344 \\
\hline 2 & 50 & 100 & 5 & 50 & 30 & 15 & 15 & 0,4340 \\
\hline 3 & 50 & 100 & 5 & .50 & 30 & 15 & 30 & 0,4342 \\
\hline 4 & 50 & 100 & 5 & 50 & $30^{\circ}$ & 15 & 5 & $0,4344^{\prime}$ \\
\hline 5 & 50 & 200 & 5 & $\therefore 50$ & 30 & 15 & 15 & 0,4346 \\
\hline 6 & $50^{\circ}$ & 300 & 5 & -50 & 30 & 15 & 15 & $0,434 \mathrm{3}$ \\
\hline 7 & 50 & .50 & 5 & 50 & 30 & 15 & rasch & 0,4344 \\
\hline 8 & 50 & 100 & 5 & 50 & 30 & 15 & & 0,4347 \\
\hline 9 & $\therefore 50$ & 200 & 5 & 50 & $\quad 30$ & 15 & & 0,4348 \\
\hline 10 & 50 & 300 & 5 & 50 & $3: 30$ & 15 & 4 & 0,4346 \\
\hline
\end{tabular}


- Bei sämtlichen Versuchen aus dieser Tabelie ist die Zusammensetzung des Fällungsmittels gleich. Sie unterscheiden sich durch die Yerdịnnung der Fällungslösung und durch die Füllungsdauer. Man ersicht aus dieser Tabelle 1., dass, wen die, Fälung langsain ausgeführt wird, man für das Magnesiumpyrophosphat fast gleiche Zahlen erhäl, abgesehen ron der Konzentration und von der Fällungsdauer (Versuche 1 bis 6); 2., dass die Zahlen für das Magnesiumpyrophosphat bei den raschen Fällungen (Versuche 7 bis 10) gleichfalls unabhängig ron der Konzentration der Fällungslosung und innerhalb der Grenzen der Versuchsébler gleich denjenigensind, welche bei langsamem Fallen erhatten werden.

\section{Tabelle 2.}

Langsame. Fällungen bei Gegenwart verschiedener: Mengen Magnesiumchlorid, Ammoniumchlorid, Kaliumellorid asw.

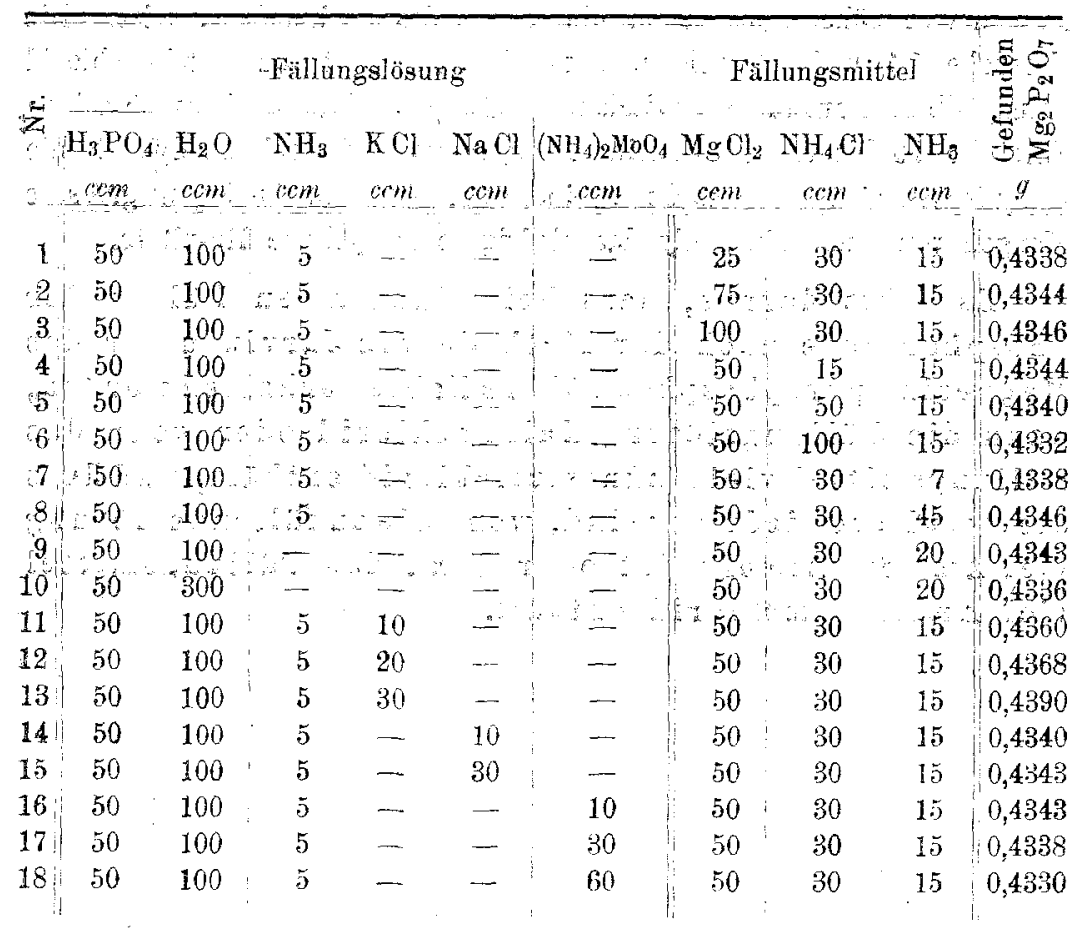

Bomerkung. Bei sämtlichen Versuchen aus dieser Tabello ist die Fâllungsdauer 15 Min. 
Die Versuche 1 bis 3 sind ausg̉efühirt, um den Einflủss des Magnesiumchlorids auf den Fällungsvorgang zu erforschen. Ihre Resultate zeigen, dass die Zahlen für das Magnesiumpyrophosphat zunehmen, wenn die Menge des Magnesiumchlorids zunimmt. Die Versuche 4 bis 6 bezweckten, den Einfluss des Ammoniumchlorids zu untersuchen. Aus ihren Resultaten ist $\mathrm{zu}$ ersehen, dass die Menge des Magnesiumpyrophosphats abnimmt, wenn die Menge des Ammoniumchlorids zunimmt. Die Versuche 7 bis 8 sind ausgeführt, um den Einfluss des Ammoniaks zu erforschen. Ihre Resultate zeigen, dass für das Magnesiumpyrophosphat höhere Zahlen erhalten werden, wenn die Menge des Ammoniaks grösser ist. Bei den Versuchen 9 und 10 enthält die Fällungsiösung kein Ammoniak. Thre Resultate zeigen, dass dieser Umstand die analytischen Resultate nicht wesentlich beeinflusst. Versuche 11 bis 13 sind ausgeführt, um den Einfluss von Kaliumchlorid auf die analytischen Resultate $\mathrm{zu}$ erforschen. Aus ihren Resultaten ist zu ersehen, dass die Gegenwart von Kaliumchlorid höhere Zahlen für das Magnesiumpyrophosphat bedingt und zwar um so höhere, je grösser die jMenge des Kaliumchlorids ist. Die Versuche 14 und 15 zeigen, dass die Gegenwart von Natriumchlorid keinen Einfluss auf die analytischen Resultate ausübt. Schliesslich zeigen Versuche 16 bis 18 , dass kleine Mengen von Ammoniummolybdat die analytischen Resultate nicht beeinflussen, dagegen bedingen grössere Mengen von Ammoniummolybdat niedrigere analytische Resultate.

Es folgt daraus, dass beim langsamen Fällen der Phosphorsäure nach der Methode von Järvinen für das Magnesiampyrophosphat höhereZahlenerhalten werden, wenn die Fällungslösung Kaliumchlorid oder wenn das Fällungsmittel viel Magnesiumchlorid enthält, und dass die Resultate niedrigersind, wenndiezu fällende Lösung viel Ammoniummolybdat oder wenn das Fällungsmittel viel Ammoniumehlorid enthält. 
Tabelle 3.

Rasche Fällungen bei Gegenwart verschiedener Mengen Magnesiumchlorid, Ammoniumchlorid, Kaliumchloridusw.

\begin{tabular}{|c|c|c|c|c|c|c|c|c|c|c|}
\hline \multirow{2}{*}{$\dot{z}$} & \multicolumn{6}{|c|}{ Fällungslösung } & \multicolumn{3}{|c|}{ Fällungsmittel } & \multirow{2}{*}{ 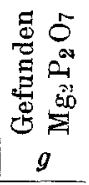 } \\
\hline & $\left|\begin{array}{c}\mathrm{H}_{3} \mathrm{PO}_{4} \\
\mathrm{ccm}\end{array}\right|$ & $\begin{array}{r}\mathrm{H}_{2} \mathrm{O} \\
\mathrm{ccm} \\
\end{array}$ & $\begin{array}{r}\mathrm{NH}_{3} \\
\mathrm{ccm} \\
\end{array}$ & $\begin{array}{l}\mathrm{KCl} \\
\mathrm{ccm}\end{array}$ & $\begin{array}{c}\mathrm{NaCl} \\
\mathrm{ccm}\end{array}$ & $\left(\begin{array}{c}\left(\mathrm{NH}_{4}\right)_{2} \mathrm{MoO}_{4} \\
\mathrm{ccm}\end{array}\right.$ & $\begin{array}{c}\mathrm{Mg} \mathrm{Cl}_{2} \\
\mathrm{ccm}\end{array}$ & $\begin{array}{c}\mathrm{NH}_{4} \mathrm{Cl} \\
\mathrm{ccm}\end{array}$ & $\begin{array}{l}\mathrm{NH}_{3} \\
\mathrm{ccm}\end{array}$ & \\
\hline 1 & 50 & 100 & 5 & - & - & - & 25 & 30 & 15 & 0,4344 \\
\hline 2 & 50 & 100 & 5 & - & - & - & 50 & 30 & 15 & 0,4348 \\
\hline 3 & 50 & 100 & 5 & - & - & - & 100 & 30 & 15 & 0,4358 \\
\hline 4 & 50 & 100 & 5 & - & 一 & -- & 50 & 15 & 15 & 0,4353 \\
\hline 5 & 50 & 100 & 5 & $\ldots$ & - & - & 50 & 50 & 15 & 0,4347 \\
\hline 6 & 50 & 100 & 5 & - & - & - & 50 & 100 & 15 & 0,4346 \\
\hline 7 & 50 & 100 & 5 & - & - & - & 50 & 30 & 7 & 0,4344 \\
\hline 8 & 50 & 100 & 5 & - & - & - & 50 & 30 & 45 & 0,4347 \\
\hline 9 & 50 & 100 & 5 & 10 & - & - & 50 & 30 & 15 & 0,4370 \\
\hline 10 & 50 & 100 & 5 & 30 & - & -.- & 50 & 30 & 15 & 0,4406 \\
\hline 11 & 50 & 100 & 5 & - & 10 & - & 50 & 30 & 15 & 0,4348 \\
\hline 12 & 50 & 100 & 5 & - & 30 & - & 50 & 30 & 15 & 0,4347 \\
\hline 13 & 50 & 100 & 5 & - & - & 10 & 50 & 30 & 15 & 0,4349 \\
\hline 14 & 50 & 100 & 5 & - & - & 30 & 50 & 30 & 15 & 0,4348 \\
\hline 15 & 50 & 100 & 5 & - & - & 60 & 50 & 30 & 15 & 0,4342 \\
\hline
\end{tabular}

Die Versuche aus dieser Tabelle unterscheiden sich von den entsprechenden Versuchen der Tabelle 2 nur dadurch, dass hier alle Fällungen rasch ausgeführt sind. Der Einfluss, welchen verschiedene Mengen von Magnesiumchlorid, Ammoniumchlorid, Kaliumchlorid usw, auf die analytischen Resultate ausüben, ist hier derselbe, wie bei den Versuchen aus der Tabelle 2. Der wesentliche Unterschied $z$ wischen den Resultaten der langsamen und der raschen Fällungen nach der Methode von Järvinen besteht darin, dass bei den raschen Fällungen für das Magnesiumpyrophosphat überhauptetwas höhere Zahlen erhalten werden, alswenn die Fällung unter denselben Versuchsbedingungen langsam ausgeführt wird.

2. Versuche nach der Methode von Jörgensen.

Diese Versuche sind mit denselben Lösungen und denselben Mengen ausgeführt, wie die Versuche nach der Methode von Järvinen. Sie unterscheiden sich von den letzten nur dadurch, dass die Zusammensetzung der Fällungslösung und des Fällungsmittels verschieden sind. 
Tabelle 4.

Einfluss der Konzentration und der Fällungsdauer.

\begin{tabular}{|c|c|c|c|c|c|c|c|}
\hline $\mathrm{Nr}$ & $\begin{array}{r}\mathrm{H}_{3} \mathrm{PO}_{4} \\
\mathrm{ccm} \\
\end{array}$ & $\begin{array}{r}\text { Fallu } \\
\mathrm{H}_{2} \mathrm{O} \\
\text { crm } \\
\end{array}$ & $\begin{array}{c}\mathrm{NH}_{4} \mathrm{Cl} \\
\mathrm{ecm} \\
\end{array}$ & $\begin{array}{l}\mathrm{NH}_{3} \\
c \mathrm{~cm} \\
\end{array}$ & $\begin{array}{c}\text { Fallungs- } \\
\text { mittel } \\
\mathrm{MgCl}_{2} \\
\mathrm{ccm}\end{array}$ & $\begin{array}{l}\text { Fällungs- } \\
\text { daner }\end{array}$ & $\begin{array}{c}\text { Gefunden } \\
\mathrm{Mg}_{2} \mathrm{P}_{\mathbf{2}} \mathrm{O}_{7} \\
g\end{array}$ \\
\hline 1 & & 50 & 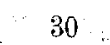 & 20 & 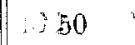 & 15 Win. & 0,4 \\
\hline 2 & 50 & $\quad 100$ & 30 & 20 & 50 & 30 & 0,4 \\
\hline 3 & 50 & 100 & 30 & $20 \cdots$ & 50 & 15 & 0 \\
\hline 4. & 5 & $\therefore 100$ & 30 & 20 & 5 & 5 & \\
\hline 5 & 5 & $\because 200$ & 30 & 20 & $\check{0}$ & $" 15$ & \\
\hline 6 & 50 & $\cdots=300$ & 30 & 20 & 5 & 15 & 0.4346 \\
\hline$\therefore 7$ & 50 & $\therefore 50$ & 30 & 20 & 5 & rasch & 0,4344 \\
\hline $8 \therefore$ & 50 & 100 & 30 & 20 . & 5 & 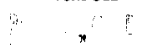 & $0,4346^{\circ}$ \\
\hline 9 & 5 & 200 & 30 & 20 & 50 & & 0,4348 \\
\hline 10 & 50 & 300 & 30 & 20 & 50 & & 0,4344 \\
\hline
\end{tabular}

Die einzelnen Versuche aus dieser Tabelle unterscheiden sich nur durch die Konzentration der Fällungslösung und durch die Fällungșdaver. A us ihren Resultaten ist $z$ u erselien, dass für das. Magnesiumpyrophosphat fast gleiche Zahlen erhalten werden, gleichgaltig, wie stark die Verdünung der Fallungslösung und wie gross die Fällungsdauer ist.

Tabelle 5 .

Langsame Fällungen bei Gegenwart verschiedener Mengen Magnesiumchlorid, Ammoniumchlorid, Kaliumehlorid usw.

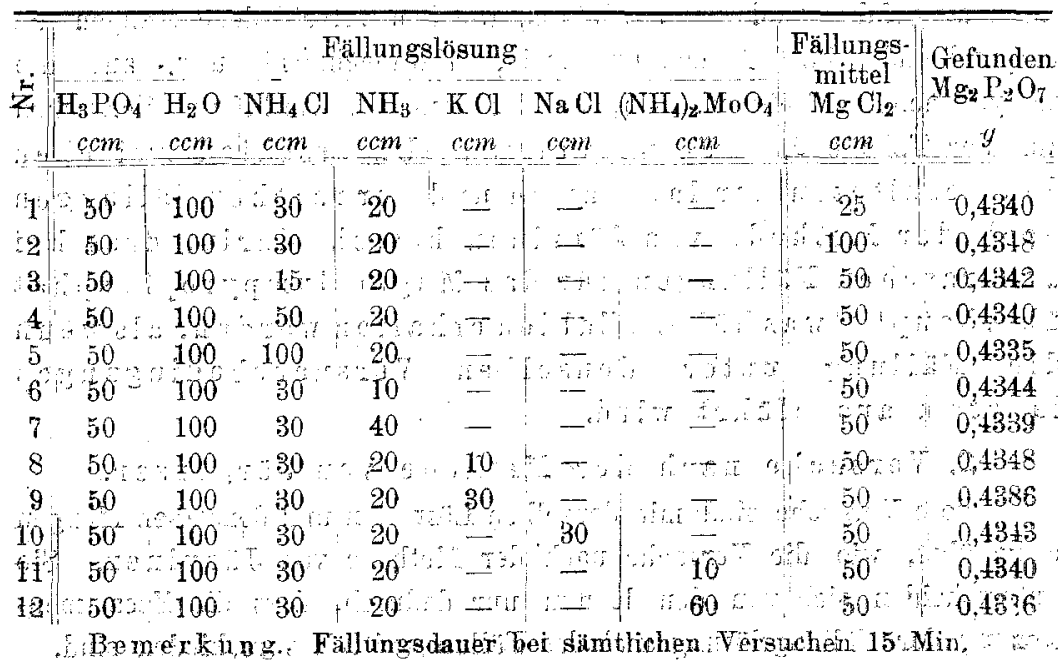


Es geht aus dieser Tabelle hervor, dass beim langsamen Fällen nach der Methodevon Jörgensen die Zahlen für das Magnesiumpyrophosphat höher sind, wenn die zu fällende Lösung Kaliumchlorid enthält (Versuche 8 und 9) oder wenn die Mengedes Magnesiamchloridsim Fällungsmittel gross ist (Versuch 2). Dagegen erhält man für das Magnesiumpyrophosphat niedrigere Zahlen, wenn die zu fällende Lösung grosse Mengen von Ammoniumchlorid oder Ammoniummolybdat enthält (Versuche 4, 5, 12). Die Gegenwart von Natriumchlorid und die Menge des Ammoniaks in der Fällungslösung beeinflusst die analytischen Resultate nicht stark (Versuche 6, 7 und 10).

Tabelle 6.

Rasche Fällnngen bei Gegenwart verschiedener Mengen Magnesiumchlorid, Ammoniumchlorid, Kaliumchlorid usw.

\begin{tabular}{|c|c|c|c|c|c|c|c|c|c|}
\hline \multirow[b]{2}{*}{ 等 } & \multicolumn{7}{|c|}{ Fällungslösung } & \multirow{2}{*}{$\mid \begin{array}{c}\text { Fällungs- } \\
\text { mittel } \\
\mathrm{Mg} \mathrm{Cl} \\
\text { ccm }\end{array}$} & \multirow{2}{*}{$\begin{array}{c}\text { Gefunden } \\
\mathrm{Mg}_{2} \mathrm{P}_{2} \mathrm{O}_{7} \\
g \\
\end{array}$} \\
\hline & {$\left[\begin{array}{c}\mathrm{H}_{3} \mathrm{PO}_{4} \\
\mathrm{ccm}\end{array}\right.$} & $\begin{array}{l}\mathrm{H}_{2} \mathrm{O} \\
\mathrm{ccm} \\
\end{array}$ & $\begin{array}{c}\mathrm{HN}_{4} \mathrm{CI} \\
\text { ccm }\end{array}$ & $\begin{array}{l}\mathrm{NH}_{3} \\
\mathrm{ccm}\end{array}$ & $\begin{array}{l}\mathrm{KCl} \\
\mathrm{ccm}\end{array}$ & $\begin{array}{c}\mathrm{NaCl} \\
\mathrm{ccm}\end{array}$ & $\begin{array}{c}\left(\mathrm{NH}_{4}\right)_{2} \mathrm{MoO}_{4} \\
\mathrm{ccm}\end{array}$ & & \\
\hline 1 & 50 & 100 & 30 & 20 & - & - & - & 25 & 0,4346 \\
\hline 2 & 5 & 100 & 30 & 20 & - & - & - & 100 &, 4362 \\
\hline 3) & 5 & 100 & 15 & 20 & - & - & - & 50 & 0,4346 \\
\hline $4 \|$ & .50 & 10 & 16 & 20 & - & - & - & 50 & 0,4336 \\
\hline 5 & 50 & 100 & 3 & 10 & - & - & - & 50 & 0,4346 \\
\hline 6 & 50 & 100 & 34 & 44 & - & - & - & 50 & 0,4350 \\
\hline 7 & 50 & 10 & 30 & 20 & 1 & -_- & $\ldots$ & 50 & 0,4374 \\
\hline 8." & 50 & 10 & 30 & 20 & 30 & - & - & 50 & 0,4400 \\
\hline $9 \|$ & 50 & 10 & 30 & 2 & - & 30 & - & 50 & 0,4348 \\
\hline 0 & 50 & 100 & 36 & 20 & - & - & 10 & 50 & 0,4347 \\
\hline 11 . & 50 & 100 & 30 & 20 & - & - & 60 & 50 & 0,4333 \\
\hline
\end{tabular}

Man ersieht aus dieser Tabelle: 1, dass die Zahlen für das Magnesiumpyrophosphat höher sind, wenn die Menge des Fälungsmittels gross ist (Versuche 1 und 2); 2, dass die Zahlen für das Magnesiumpyrophosphat niedriger sind, wenn die Menge des Ammoniumchlorids oder des Ammoniummolybdats in der Fällungslösung gross ist (Versuche 3,4,10,11); 3, dass die analytischen Resultate hoch sind, wenn die Fällangslösung Kaliumchlorid enthält, und, 4 , dass die Gegenwart von verschiedenen Mengen von Ammoniak oder Natrjumchlorid die analytischen Resultate nicht merklich beeinflusst.

Fresenius, Zeitschrift P. anal. Chemie. LVII. Jahrgang. 11. u. 12. Heft. 33 e 
Vergleicht man die Resultate der Tabellen 5 und 6 , so sieht man, dass die Menge des Fällungsmittels und auch die Gegenwart verschiedener Mengen von Ammoniumchlorid, Kaliumchlorid, Ammoniummolybdat usw. in ähnlicher Weise die Zahlen für das Magnesiumpyrophosphat beeinflussen. Der wesentliche Unterschied zwischen den Resultaten der Tabellen 5 and 6 , oder zwischen den Resultaten der langsamen und der raschen Fällung besteht darin, dass die Zahlen für das Magnesiumpyrophosphat etwas höher sind, wenn die Fällung rasch ausgeführt wird. Vergleicht man andererseits die Resultate der Versuche aus den Tabellen 1 bis 6, oder die Bestimmungen nach der Methode von Järvinen mit denjenigen nach der Methode von Jörgensen, so kommt man zu dem Schluss, dass unter gleichen Versuchsbedingungen für das Magnesiumpyrophosphat innerhalb der Grenzen der Versuchsfehler gleiche Zahlen erhalten werden. Das heisst, dass unter sonst gleichen Bedingungen der Fällungsvorgang auf gleiche Weise verläuft, gleichgültig, ob die Fällung nach der Methode von Järvinen oder nach der Methode von Jörgensen ausgeführt wird.

3. Versuche nach der Methode von Schmitz.

Bei der Ausführung dieser Versuche wurden dieselben Bedingungen beibehalten. Sie unterscheiden sich von den vorigen nur durch die Zusammensetzung der zu fällenden Lösung und des Fällungsmittels.

\section{Tabelle 7.}

Einfluss der Konzentration und der Fällungsdauer.

\begin{tabular}{|c|c|c|c|c|c|c|c|c|c|}
\hline \multirow[b]{2}{*}{ 息 } & \multicolumn{5}{|c|}{ Fällungslösung } & \multicolumn{2}{|c|}{ Fällungsmittel } & \multirow{2}{*}{$\begin{array}{c}\text { Fällungs } \\
\text { dauer }\end{array}$} & \multirow{2}{*}{$\begin{array}{c}\text { Gefunden } \\
\mathrm{Mg}_{2} \mathrm{P}_{2} \mathrm{O}_{7} \\
g\end{array}$} \\
\hline & $\begin{array}{c}\mathrm{H}_{3} \mathrm{PO}_{4} \\
\mathrm{ccm}\end{array}$ & $\begin{array}{r}\mathrm{H}_{2} \mathrm{O} \\
\mathrm{ccm}\end{array}$ & $\begin{array}{c}\mathrm{NH}_{4} \mathrm{Cl} \\
\mathrm{ccm}\end{array}$ & $\begin{array}{c}\mathrm{MgCl}_{2} \\
\mathrm{ccm}\end{array}$ & $\begin{array}{l}\mathrm{HCl} \\
\mathrm{ccm}\end{array}$ & $\begin{array}{c}\text { I. } \mathrm{NH}_{3} \\
\mathrm{ccm}\end{array}$ & $\begin{array}{c}\text { II. } \mathrm{NH}_{3} \\
\mathrm{~cm}\end{array}$ & & \\
\hline 1 & 50 & 50 & 30 & 50 & 1 & 50 & 20 & $15 \mathrm{Min}$. & 0,4346 \\
\hline 2 & 50 & 100 & 30 & 50 & 1 & 50 & 20 & 5 & 0,4340 \\
\hline 3 & 50 & 100 & 30 & 50 & 1 & 50 & 20 & 15 & 0,4342 \\
\hline 4 & 50 & 100 & 30 & 50 & 1 & 50 & 20 & & 0,4344 \\
\hline 5 & 50 & 200 & 30 & 50 & 1 & 50 & 20 & & 0,4339 \\
\hline 6 & 50 & 300 & 30 & 50 & 1 & 50 & 20 & 15 & 0,4337 \\
\hline 7 & 50 & 50 & 30 & 50 & 1 & 50 & 20 & rasch & 0,4330 \\
\hline 8 & 50 & 100 & 30 & 50 & 1 & 50 & 20 & . & 0,4332 \\
\hline 9 & 50 & 200 & 30 & 50 & 1 & 50 & 20 & n & 0,4335 \\
\hline 10 & 50 & 300 & 30 & 50 & 1 & 50 & 20 & " & 0,4330 \\
\hline
\end{tabular}

Bemerkung. In dieser wie in den folgenden Tabellen bedeuten die Zahlen unter $\mathrm{I}$. $\mathrm{NH}_{3}$ die $c c m$ 2,5\% $/ 0$ iges Ammoniak und die Zahlen unter II. $\mathrm{NH}_{3}$ die $\mathrm{ccm}$ konzentriertes Ammoniak. Die für diese Versuche angewangte Salzsäure ist 6-normal. 
Aus den Versuchen 1 bis 6 ist zu ersehen, dass für das Magnesiumpyrophosphat niedrigere Zahlen erhalten werden, wenn die Fällungslösung stärker verdünnt ist. Die Versuche 7 bis 10 zeigen, dass die Zahlen für das Magnesiumpyrophosphat niedriger.sind, wenn die Fällung rasch ausgeführt wird.

Tabelle 8.

Langsame Fällungen bei Gegenwart verschiedener Mengen Magnesiumchlorid, Ammoniumchlorid, Kaliumchlorid usw.

\begin{tabular}{|c|c|c|c|c|c|c|c|c|c|c|}
\hline \multirow{2}{*}{$\dot{\mathbf{Z}}$} & \multicolumn{7}{|c|}{ Fällungslösung } & \multicolumn{2}{|c|}{ Fällungsmittel } & \multirow{2}{*}{ 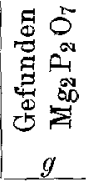 } \\
\hline & $\begin{array}{c}\mathrm{H}_{3} \mathrm{PO}_{4} \\
\mathrm{ccm}\end{array}$ & $\begin{array}{r}\mathrm{H}_{2} \mathrm{O} \\
\text { ccm } \\
\end{array}$ & $\mid \begin{array}{c}\mathrm{NH}_{4} \mathrm{CI} \\
\mathrm{ccm}\end{array}$ & $\left|\begin{array}{c}\mathrm{Mg} \mathrm{Cl}_{2} \\
\mathrm{ccm}\end{array}\right|$ & $\begin{array}{l}\mathrm{K} \mathrm{Cl} \\
\mathrm{ccm}\end{array}$ & $\begin{array}{c}\mathrm{NaCl} \\
c c m\end{array}$ & $\left(\begin{array}{c}\left(\mathrm{NH}_{4}\right)_{2} \mathrm{MnO}_{4} \\
\mathrm{cem}\end{array}\right.$ & $\begin{array}{c}\mathrm{NH} \\
\mathrm{ccm}\end{array}$ & $\begin{array}{c}\text { II. } \mathrm{NH}_{3} \\
\mathrm{ccm}\end{array}$ & \\
\hline 1 & 50 & 100 & 30 & 25 & - & - & 一 & 30 & 20 & 0,4342 \\
\hline 2 & 50 & 100 & 30 & 100 & - & 一 & - & 30 & 20 & 0,4350 \\
\hline 3 & 50 & 100 & 20 & 50 & - & - & - & 30 & 20 & $0,43 \pm 5$ \\
\hline 4 & 50 & 100 & 100 & 50 & - & - & - & 30 & 20 & 0,4340 \\
\hline 5 & 50 & 100 & 30 & 50 & - & - & - & 30 & 10 & 0,4389 \\
\hline 6 & 50 & 100 & $\div 0$ & 50 & - & - & - & 30 & 40 & 0,4344 \\
\hline 7 & 50 & 100 & 30 & 50 & 20 & - & - & 30 & 20 & 0,4463 \\
\hline 8 & 50 & 100 & 0 & 50 & 30 & - & - & 30 & 20 & 0,4395 \\
\hline 9 & 50 & 100 & 30 & 50 & - & 30 & - & 30 & 20 & 0,4343 \\
\hline 10 & 50 & 100 & 30 & 50 & - & - & 10 & 30 & 20 & 0,4346 \\
\hline 11 & 50 & 100 & 30 & 50 & - & - & 60 & 30 & 20 & 0,4356 \\
\hline
\end{tabular}

Bemerkung. Die Fällungslösung enthielt bei allen Versuchen aus dieser Tabelle je $1 \mathrm{~cm}$ 6-normale Salzsäure. Die Fällungsdauer betrug bei sämtlichen Versuchen 15 Minuten.

Es geht aus dieser Tabelle hervor, dass die Zahlen für das Magnesiumpyrophosphat höher sind, wenn die zu fällende Lösung grössere Mengen Kaliumehlorid, Magnesiumchlorid oder Ammoniummolybdat enthält (siehe Versuche 2, 7,8,11), oder wenn die Menge des als Fällungsmittel zugesetzten konzentrierten Ammoniaks grösser ist (Versuche 4 und 5). Die Gegenwart grösserer Mengen von Ammoniumchlorid bedingt niedrigere Zahlen für das Magnesiumpyrophosphat (Versuche 3 und 4) und die Gegenwart von Natriumchlorid übt keinen merklichen Einfluss auf die analytischen Resultate aus. 
Zum Verständnis der Tatsache, dass die Gegenwart von Ammoniummolybdat höhere Zahlen für das Magnesiumpyrophosphat bedingt, muss folgendes berücksichtigt werden. Da die zu fällende Lösung sauer ist und gleichzeitig Phosphorsäure and Ammoniummolybdat enthält, fällt Ammoniumphosphormolybdat aus. Beim Zusatz von Ammoniak nimmt die Menge des Niederschlages $a b$, aber bevor er noch vollständig gelöst wird, fängt die Bildung des Magnesiumammoniumphosphats an, so dass die Ursache für die höheren Resultate darin zu suchen ist, dass zu dem Niederschlag von Magnesiumammoniumphosphat Ammoniumphosphormolybdat hinzukommt. Für diese Annahme sprechen noch einige Tatsachen, die weiter unten beschrieben werden.

\section{Tabelle 9.}

Rasche Fällargen bei Gegenwart verschiedener Mengen Magnesiumchlorid, Ammoniumchlorid, Kaliumchloridusw.

\begin{tabular}{|c|c|c|c|c|c|c|c|c|c|c|}
\hline \multirow[b]{2}{*}{ 安 } & \multicolumn{7}{|c|}{ Fällungslösung } & \multicolumn{2}{|c|}{ Fällungsmittel } & \multirow{2}{*}{ 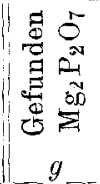 } \\
\hline & $\left|\begin{array}{c}\mathrm{H}_{3} \mathrm{PO}_{4} \\
\mathrm{ccm}\end{array}\right|$ & $\begin{array}{r}\mathrm{H}_{2} \mathrm{O} \\
\mathrm{ccm} \\
\end{array}$ & $\begin{array}{c}\mathrm{NH}_{4} \mathrm{Cl} \\
\mathrm{ccm} \\
\end{array}$ & $\begin{array}{c}\mathrm{MgCl}_{2} \\
\mathrm{ccm}\end{array}$ & $\begin{array}{l}\mathrm{KCl} \\
\mathrm{cmm} \\
\end{array}$ & $\begin{array}{r}\mathrm{NaCl} \\
\mathrm{ccm} \\
\end{array}$ & $\begin{array}{c}\left(\mathrm{NH}_{4}\right)_{2} \mathrm{M}_{0} \mathrm{O}_{4} \\
\mathrm{ccm} \\
\end{array}$ & $\begin{array}{l}\mathrm{NH}_{3} \\
\mathrm{ccm}\end{array}$ & $\begin{array}{r}\mathrm{N} . \mathrm{NH}_{3} \\
\mathrm{cem} \\
\end{array}$ & \\
\hline 1 & 50 & 10 & 30 & 25 & - & - & - & 30 & 20 & 0,4330 \\
\hline 2 & 5 & & 3 & 100 & - & - & - & 30 & 20 & 0,43 \\
\hline 3 & 5 & & & 50 & - & - & - & 30 & 20 & 0,4338 \\
\hline 4 & 5 & & 100 & 50 & - & - & - & 30 & 20 & 0,4930 \\
\hline 5 & 5 & & 3 & & - & 一 & - & 30 & 10 & 0,43 \\
\hline 6 & 5 & & 30 & 5 & - & - & - & 3 & 4. & 0,4840 \\
\hline 7 & 50 & 10 & 30 & 50 & 11 & - & - & 30 & 20 & 0,4345 \\
\hline 8 & 5 & 10 & 30 & 50 & 30 & - & - & 30 & 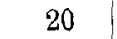 & $0,4 \cdot 38$ \\
\hline 9 & 5 & & 0 & & - & 30 & - & 30 & 20 & 0,4833 \\
\hline 10 & 5 & 10 & 30 & 50 & - & $\ldots$ & 10 & 30 & 20 & 0,4335 \\
\hline 11 & 50 & 100 & 30 & 50 & - & - & 60 & 30 & 20 & 0,4349 \\
\hline
\end{tabular}

Bemerkung. Die Fällungslösung enthielt bei sämtlichen Versuchen aus dieser Tabelle noch je $1 \mathrm{ccm}$ 6-normale Salzsäure.

Die Versuche aus dieser Tabelle zeigen: 1. dass die Gegenwart grosser Mengen ron Maguesiumehlorid, Kaliumchlorid und Ammoniummolybdat in der zu fällenden Lösung höhere Zahlen fär das Magnesiumpyrophosphat bedingt (s. Versuche 2, 7,8, 11); 2. dass die Zahlen für das Magnesiumpyrophosphat niedriger sind, 
wenn die Fällungslösung grosse Mengen Ammoniumchlorid enthält (Versuche 3 und 4), und höher sind, wenn die Menge des zugesetzten konzentrierten Ammoniaks gross ist.

Vergleicht man die Resultate der Versuche aus Tabelle 9 mit denjenigen aus Tabelle 8, so sieht man, dass der Unterschied $z$ wischen den Resultaten der raschen und der langsamen Fällungen hauptsächlich darin besteht, dass bei den raschen Fällungen die Zahlen für das Magnesiumpyrophosphat überhaupt etwas niedriger sind. Vergleicht man andererseits die Resultate aus den Versuchen nach der Methode von Schmitz mit denjenigen nach den Methoden von Järvinen und Jörgensen, so kommt man zu folgenden Schlüssen: 1. dass, wenn dieFällungen nach deu verschiedenen Methoden langsam und unter Einhalten von sonst gleichen Versuchsbedingungen ausgeführtwerden, man für das Magnesiumpyrophosphat fast gleiche Zablen erhält, und 2. wenn die Fällungen nach den drei Methoden rasch ausgefuhrtwerden, die Resultate der Schmitzschen Methode etwas niedriger ausfallen.

4. Übersicht über die Resultate.

Die bisher mitgeteilten Versuche erlauben uns einige Schlüsse auf den Verlauf der Vorgänge zu ziehen, welche sich beim Fällen der Phosphorsäure als Magnesiumammoniumphosphat abspielen.

Wie wir im allgemeinen Teil gesehen haben, sind beim Fällen von Phosphorsäure als Magnesiumammoniumphosphat theoretisch verschiedene sekundäre Fällungsvorgänge denkbar. Wenn sich diese Vorgänge in der Tat so abspielten, müsste man erwarten: 1. dass die analytischen Resultate der verschiedenen Methoden verschieden ausfallen und zwar 2. auch dann, wenn die Fällung nach einer bestimmten Methode, aber bei verschiedener Konzentration der daran beteiligten Stoffe ausgeführt wird.

Die Tatsachen zeigen, dass die verschiedenen Methoden zur Fällung der Phosphorsäure als Magnesiumammoniumphosphat gleiche analytische Resultate geben, wenn die Fällung unter sonst gleichen Versuchsbedingungen ansgeführt wird. (Nur wenn die Fällung nach der Methode. von Schmitz rasch geschieht, sind die Resultate etwas niedriger.) Daraus ist zu schliessen, dass beim Fällen der Phosphorsäure aus einer 
Lösung, welche nur bis zum Sieden erhitzt ist, der Hauptfällungsvorgang von keinen sekundären Fällungsvorgängen begleitet ist, gleichgültig nach welcher Methode die Fällang stattfindet.

Die Tatsache, dass die Zahlen für das Magnesiumpyrophosphat verschieden sind, wenn die Fällung nach einer bestimmten Methode, aber bei Gegenwart verschiedener Mengen der sich an dem Vorgang beteiligenden 'Stoffe geschieht, widerspricht scheinbar diesem Schlusse. Ihm widerspricht auch die Tatsache, dass die Resultate einer und derselben Methode von der Fällungsdauer abhängig sind. Bei der Erklärung dieser Widersprüche muss aber berücksichtigt werden, dass die Abweichungen der analytischen Resultate voneinander, welche durch die verschiedene Konzentration der daran beteiligten Lösungen und durch die Fällungsdauer verursacht werden, verhältnismäßig klein sind.

Zur Erklärung des Umstandes, dass beim Fällen von Phosphorsäure bei Gegenwart grosser Mengen von Ammoniumchlorid die analytischen Resultate niedrig ausfallen, kann angenommen werden: 1 . dass das Ammoniumchlorid die Bildung des sekundären Produkts $\mathrm{Mg}\left[\left(\mathrm{NH}_{4}\right)_{2} \mathrm{PO}_{4}\right]_{2}$ begünstigt und 2. dass die Löslichkeit des Magnesiumammoniumphosphats bei Gegenwart grosser Mengen von Ammoniumchlorid zunimmt. Von diesen Annahmen kann die erste nicht alle Tatsachen befriedigend erklären; dagegen ist dies mit Hilfe der zweiten vollständig der Fall. Diese Annahme stimmt vollständig nit der von K. Bube (l. c.) festgestellten Tatsache überein, dass die Löslichkeit des Magnesiumammoniumphosphats in Wasser bei Gegenwart grosser Mengen von Ammoniumchlorid zunimmt.

Auf dieselbe Weise ist auch die Tatsache zu erklären, dass grosse Mengen von Ammoniummolybdat niedrigere Zahlen für das Magnesiumpyrophosphat bewirken, wenn die Fällung nach den Methoden von Järvinen oder Jörgensen ausgeführt wird. Qualitative Versuche zeigen in der Tat, dass das Magnesinmammoniumphosphat durch eine Lösung von Ammoniummolybdat ziemlich leicht gelöst wird. Die Gegenwart von Ammoniak oder von Magnesiumchlorid vermindert zwar die Löslichkeit des Magnesiumammoniumphosphats, aber ganz beseitigt wird sie nicht.

Auch die Tatsache, dass grosse Mengen von Magnesiumchlorid höhere analytische Resultate verursachen, kann auf zweierlei Weise erklärt werden! 1. durch sekundäre Fällungsvorgänge und 2. durch Abnahme der Löslichkeit des Magnesiumammoniumphosphats. Wie im allgemeinen Teil auseinandergesetzt wurde, sind beim Fällen von Phosphor- 
säure als Magnesiumammoniumphosphat verschiedene sekundären Fällungsvorgänge möglich, welche höhere analytische Resultate hervorrufen können. Doch sind die Versuchsergebnisse in vorliegender Arbeit durch diese Vorgänge nicht befriedigend zu erklären. Dagegen bietet dies keine Schwierigkeiten, wenn angenommen wird, dass bei Gegenwart von Magnesiumchlorid die Löslichkeit des Magnesiumammoniumphosphats abnimmt. Diese Annahme stimmt mit der von $\mathrm{K}$. Bube (l. c.) festgestellten Tatsache überein, dass die Löslichkeit des Magnesiumammoniumphosphats bei Gegenwart von Magnesiumchlorid abnimmt.

Auch die Tatsache, dass ein grosser Ammoniaküberschuss höhere analytische Resultate bedingt, ist durch die Löslichkeit des Magnesiumammoniumphosphats befriedigend erklärlich.

Es folgt aus alledem, dass die Abweichungen, welche bei dem analytischen Resultate beobachtet werden, wenn die Fällung der Phosphorsäure als Magnesiumammoniumphosphat unter verschiedenen Bedingungen erfolgt, mit der Löslichkeit des Niederschlages leicht erklärt werden können. Diese Auffassung wird noch wahrscheinlicher, wenn man berücksichtigt, dass die Löslichkeit des Magnesiumammoniumphosphats in Wasser ziemlich gross ist (s. K. Bube [l. c ]) und dass der Einfluss der verschiedenen Verbindungen, bei deren Gegenwart es entsteht, auf seine Löslichkeit ein verschiedener ist.

Eine Besonderheit stellt die Tatsache dar, dass die Gegenwart von Kaliumchlorid höhere analytische Resultate bedingt, ohne Rücksicht darauf, nach-welcher Methode gefällt wird. Diese Tatsache kann nicht mit der Löslichkeit des Magnesiumammoniumphosphats erklärt werden. Hierfür ist es notwendig, anzunehmen, dass die Gegenwart von Kaliumchlorid in der zu fällenden Lösung sekundäre Fällungsvorgänge bewirkt. Um die Natur dieser Vorgänge zu verstehen, müssen folgende Tatsachen berücksichtigt werden: 1. Bei Gegenwart einer und derselben Menge von Kaliumchlorid sind die analytischen Resultate nach den verschiedenen Methoden ungefähr gleich; 2. die Resultate sind höher, wenn die Menge des Kaliumchlorids grösser ist; 3. das Magnesiumpyrophosphat, welches nach dem Fällen der Phosphorsäure bei Gegenwart von Kaliumchlorid erhalten wird, enthält kein Kaliumchlorid, trotzdem sein Gewicht grösser ist, als wenn die Fällung nicht bei Gegenwart von Kaliumchlorid geschieht ${ }^{1}$ ).

1) Das ist leicht festzustellen dadurch, dass man solches Magnesiumpyrophosphat in Salpetersäure löst und mit Silbernitrat prüft. 
Hiervon ausgehend kommt man zu dem Schluss, dass die Vorgänge, welche beim Fällen von Phosphorsäure bei Gegenwart von Kaliumchlorid verlaufen, wahrscheinlich folgende sind. In der Lösung, welche gleichzeitig Ammoniumphosphat oder Phosphorsäure und Kaliumchlorid enthält, verlaufen Gleichgewichtsreaktionen, infolgedessen Ionen entstehen, welche gleichzeitig Kalium und das Radikal der Phosphorsäure enthalten (z. B. $\mathrm{KPO}_{4}{ }^{\prime \prime}$ oder $\mathrm{K}_{2} \mathrm{PO}_{4}{ }^{\prime}$ ). Wird die Anwesenbeit von solchen Anionen in der zu fällenden Lösung angenommen, so lasselı sich obige Tatsachen leicht dadurch erklären, dass diese Anionen mit den Kationen des Magnesiums schwerlösliche Verbindungen bilden. Z. B.:

$$
\mathrm{K} \mathrm{PO}_{4} "+\mathrm{Mg}{ }^{*}=\mathrm{Mg} \mathrm{KPO}_{2} \text {. }
$$

Der Umstand, dass das Natriumchlorid nicht denselben Einfluss auf die analytischen Resultate ausübt, wie das Kaliumchlorid; beweist, dass die entsprechenden Natriumverbindungen leichter löslich sind.

Die Verbindungen $\mathrm{Mg} \mathrm{KPO}_{4}$ und $\mathrm{MgNaPO}_{4}$ sind dargestellt. Auch andere Phosphate sind dargestellt worden, welche gleichzeitig Magnesium und Kalium oder Natrium enthalten ${ }^{1}$ ). Die Eigenschaften dieser Stoffe sind noch nicht gut bekannt. Die Untersuchungen von Berthelot ${ }^{2}$ ) sprechen zugunsten der Auffassung, dass beim Fällen der Phosphorsäure bei Gegenwart von Kaliumchlorid das sekundäre Produkt $\mathrm{MgK} \mathrm{PO}_{4}$ entsteht.

\section{Zweite Versuchsreihe.}

\section{B. Während der Fällung der Phosphorsäure wird die Lösung ununterbrochen gekocht.}

Bei dieser Versuchsreibe wurde die zu fällende Lösung zum Kochen erhitzt und das Fällungsmittel, ohne das Erwärmen zu unterbrechen, zugesetzt. Bei den langsamen Fällungen betrug die Fällungsdauer 15. Minuten. Bei den raschen Fällungen wurde das Fällungsmittel möglichst schnell zugesetzt und alsdann der Niederschlag mit der Flüssigkeit, aus der er entstanden war, noch 15 Min. gekocht. Die weitere Verarbeitung der Niederschläge erfolgte wie bei den Versuchen der ersten. Versuchsreihe.

1) Gmelin-Krauts, Handbuch der anorgan. Chemie II, 2, S. 487.

2) Compt. rend. 133, 1901 und Annales de chimie et de physique, 7 Serie, T. 25, I, S. 176 (1902). 
1. Versuche nach der Methode von Järrinen.

Tabelle 10.

\begin{tabular}{|c|c|c|c|c|c|c|c|c|}
\hline \multirow{2}{*}{$\mathrm{Nr}$} & \multicolumn{3}{|c|}{ Fällungslösung } & \multicolumn{3}{|c|}{ Fällungsmittel } & \multirow{2}{*}{$\begin{array}{c}\text { Fällungs- } \\
\text { dauer }\end{array}$} & \multirow{2}{*}{$\begin{array}{c}\text { Gefunder } \\
\mathrm{Ng}_{2} \mathrm{P}_{2} \mathrm{O}_{\tau} \\
g\end{array}$} \\
\hline & $\begin{array}{c}\mathrm{H}_{3} \mathrm{PO}_{4} \\
\mathrm{ccm}\end{array}$ & $\begin{array}{r}\mathrm{H}_{2} \mathrm{O} \\
\mathrm{ccm} \\
\end{array}$ & $\begin{array}{l}\mathrm{NH}_{3} \\
\mathrm{ccm} \\
\end{array}$ & $\begin{array}{c}\mathrm{MgCl}_{2} \\
\mathrm{ccm} \\
\end{array}$ & $\begin{array}{c}\mathrm{NH}_{4} \mathrm{Cl} \\
\mathrm{ccm} \\
\end{array}$ & $\begin{array}{l}\mathrm{NH}_{3} \\
\mathrm{ccm} \\
\end{array}$ & & \\
\hline 1 & 50 & 50 & 5 & 50 & 30 & 15 & $15 \mathrm{Min}$. & 0,4362 \\
\hline 2 & 50 & 100 & 5 & 50 & 30 & 15 & 15 & 0,4370 \\
\hline 3 & 50 & 200 & 5 & 50 & 30 & 15 & & 0,4376 \\
\hline 4 & 50 & 300 & 5 & 50 & 30 & 15 & & 0,4380 \\
\hline 5 & 50 & 200 & 5 & 50 & 60 & 15 & 15 & 0,4370 \\
\hline 6 & 50 & 200 & 5 & 50 & 90 & 15 & 15 & 0,4365 \\
\hline 7 & 50 & 100 & 5 & 50 & 30 & 15 & rasch & 0,4400 \\
\hline 8 & 50 & 300 & 5 & 50 & 30 & 15 & 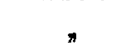 & 0,4387 \\
\hline 91 & 50 & 100 & 5 & 50 & 30 & 15 & n & 0,4345 \\
\hline
\end{tabular}

Bemerkung. Der Versuch 9 unterscheidet sich von den Versuchen $T$ und 8 dadurch, dass bei ihm nach der Fällung nicht gekocht wurde.

Die Versuche 1 bis 4 zeigen, dass für das Magnesiumpyrophosphat höhere Zahlen erhalten werden, wenn diezu fällende Lösung stärker verdünt ist. Die Versuche 5 und 6 unterscheiden sich voneinander und von Versuch 3 nur durch die Menge des Ammoniumchlorids. Aus. ihren Resultaten ist zu sehen, dass für das Magnesiumpyrophosphat niedrigere Zahlen erhalten werden, wenn. die Menge des Ammoniamchlorids grösser ist. Die Versuche 7 und 8 zeigen, dass wenn die Fällung raschausgeführt wird und der Niederschlag 15 Minuten mit der Flüssigkeit, aus der er entstanden ist, gekochtwird, für das Magnesiumpyrophosphat höhere Zahlen erhalten. werden, als wenn unter denselben Bedingungen die Fällung langsam ausgeführt wird. Zuletzt zeigt der Versuch 9, dass wenn nach der raschen Fällung der. Niederschlag nicht gekocht wird, man für das Magnesiumpyrophosphat eine viel niedrigere Zahl erhäl, als wenn. nach der Fällung gekocht wird:

Vergleicht man die Resultate aus der Tabelle 10, einerseits and die ausden Tabellen 1, 2 und 3 andererseits, so sieht man, dass wenn die Fälung nach der. 
Methodevon Järvinen so ausgeführtwird, dass während der Fällung die Lôsung ununterbrochen gekocht wird, man für das Magnesiumpyrophosphat höhere Zahlen erhält, als wenn die zu fällende Lösung unter sonst gleichen Versuchsbedingungen nur bis zum beginnenden Sieden erwärmt wird und die Fällung ohne weitere Erhitzung ausgeführt wird. Es folgt daraus, dass die Temperatur, bei welcher die Fällung nach der Methode von Järvinen ausgeführt wird, eine wichtige Bedingung für die Zuverlässigkeit der analytischen Resultate ist.

2. Versuche nach der Methode von Jörgensen.

Tabelle 11.

\begin{tabular}{|c|c|c|c|c|c|c|c|}
\hline \multirow{2}{*}{ Nr. } & \multicolumn{4}{|c|}{ Fällungslösung } & \multirow{2}{*}{$\begin{array}{c}\text { Fällungs- } \\
\text { mittel } \\
\mathrm{Mg} \mathrm{Cl}_{2} \\
\text { ccm }\end{array}$} & \multirow{2}{*}{$\begin{array}{c}\text { Fällungs- } \\
\text { dauer }\end{array}$} & \multirow{2}{*}{$\begin{array}{c}\text { Gefunden } \\
\mathrm{Mg}_{2} \mathrm{P}_{2} \mathrm{O}_{7} \\
g\end{array}$} \\
\hline & $\begin{array}{c}\mathrm{H}_{3} \mathrm{PO}_{4} \\
\mathrm{ccm} \\
\end{array}$ & $\begin{array}{l}\mathrm{H}_{2} \mathrm{O} \\
\mathrm{ccm} \\
\end{array}$ & $\begin{array}{c}\mathrm{NH}_{4} \mathrm{Cl} \\
\mathrm{ccm} \\
\end{array}$ & $\begin{array}{l}\mathrm{NH}_{3} \\
\mathrm{ccm}\end{array}$ & & & \\
\hline 1 & 50 & 50 & 30 & 20 & 50 & langsam & 0,4360 \\
\hline 2 & 50 & 100 & 30 & 20 & 50 & $\pi$ & 0,4372 \\
\hline 8 & 50 & 200 & 30 & 20 & 50 & 》 & 0,4378 \\
\hline 4 & 50 & 300 & 30 & 20 & 50 & $n$ & 0,4383 \\
\hline 5 & 50 & 200 & 60 & 20 & 50 & , & 0,4370 \\
\hline 6 & 50 & 200 & 90 & 20 & 50 & , & 0,4362 \\
\hline 7 & 50 & 100 & 30 & 20 & 50 & rasch & 0,4468 \\
\hline 8 & 50 & 300 & 30 & 20 & 50 & n & 0,4478 \\
\hline 9 & 50 & 100 & 30 & 20 & 50 & $\pi$ & 0,4348 \\
\hline
\end{tabular}

Die Versuche aus dieser Tabelle unterscheiden sich von den entsprechenden Versuchen aus Tabelle 10 nur durch die Art der Fällung. Die Resultate der Versuche bei beiden Tabellen zeigen eine gleiche Regelmäßiglreit. Die Differenz zwischen den Werten für das Magnesiumpyrophosphat bei den entsprechenden Versuchen bei beiden Tabellen liegt sogar innerhalb der Feblergrenze. Nur bei den Versuchen 7 und 8 aus Tabelle 11 wurden für das Magnesiumpyrophosphat bedeutend höhere Zahlen erhalten, als für dieselben Versuche aus Tabelle 10.

Die Versuche nach der Methode von Jörgensen zeigen also auch, dass wenn wäbrend der Fällung die Lösung beständig gekocht wird, oder wenn der durch rasche Fällung erhaltene Niederschlag von Mlagnesiumammonium- 
phosphat mit der Flüssigkeit, aus der er entstanden ist: gekocht wird, die analytischen Resultate bedeutend hober ausfallen, als wenndie zu fällende Lösung während der Fällung nicht gekochtwird, oder wenn der Niederschlag von Magnesiumamoniumphosphat nicht mit der Flüssigkeit gekocht wird, aus der er entstanden ist.

3. Versuche nach der Methode von Schmitz.

Tabelle 12.

\begin{tabular}{|c|c|c|c|c|c|c|c|c|}
\hline \multirow{2}{*}{$\mathrm{Nr}$} & \multicolumn{4}{|c|}{ Fällungslösung } & \multicolumn{2}{|c|}{ Fällungsmittel } & \multirow{2}{*}{$\begin{array}{c}\text { Fällungs- } \\
\text { dauer }\end{array}$} & \multirow{2}{*}{$\begin{array}{c}\text { Gefunden } \\
\mathrm{Mg}_{2} \mathrm{P}_{2} \mathrm{O}_{7} \\
g\end{array}$} \\
\hline & $\begin{array}{c}\mathrm{H}_{3} \mathrm{PO}_{4} \\
c c m\end{array}$ & $\begin{array}{r}\mathrm{H}_{2} \mathrm{O} \\
\mathrm{ccm}\end{array}$ & $\begin{array}{c}\mathrm{Mg} \mathrm{Cl}_{2} \\
\mathrm{ccm}\end{array}$ & $\begin{array}{c}\mathrm{NH}_{1} \mathrm{Cl} \\
\mathrm{ccm}\end{array}$ & $\begin{array}{c}\text { I. } \mathrm{NH}_{3} \\
c c m\end{array}$ & $\begin{array}{c}\text { II. } \mathrm{NH}_{3} \\
\mathrm{ccm}\end{array}$ & & \\
\hline 1 & 50 & 50 & 50 & 30 & 50 & 20 & langsam & 0,4352 \\
\hline 2 & 50 & 100 & 50 & 30 & 50 & 20 & $\pi$ & 0,4368 \\
\hline 3 & 50 & 200 & 50 & 30 & 50 & 20 & n & 0,4996 \\
\hline 4 & 50 & 300 & 50 & 30 & 50 & 20 & $n$ & 0,4650 \\
\hline 5 & 50 & 200 & 50 & 60 & 50 & 20 & $\pi$ & 0,4360 \\
\hline 6 & 50 & 200 & 50 & 90 & 50 & 20 & $\pi$ & 0,4352 \\
\hline 7 & 50 & 100 & 50 & 30 & 50 & 20 & rasch & 0,4392 \\
\hline 8 & 50 & 300 & 50 & 30 & 50 & 20 & , & 0,4387 \\
\hline 9 & 50 & 100 & 50 & 30 & 50 & 20 & , & 0,4335 \\
\hline
\end{tabular}

Bemerkung. Bei diesen Versuchen enthielt die zu fällende Lösung noch je $1 \mathrm{ccm}$ 6-normale Salzsäure. Bei den langsamen Fällungen betrug die Fällungsdauer 15 Minuten.

Es geht aus dieser Tabelle hervor, dass die Zahlen für das Magnesiumpyrophosphat höher ausfallen, wenn die zu fällende Lösung verdünnter ist. Besonders hoch sind die Zahlen bei den Versuchen 3 und 4. Die übrigen Versuche zeigen dieselbe Abhängigkeit von den Versuchsbedingungen, wie die Versuche aus den Tabellen 10 und 11.

Die Versuche nach der Methode von Schmitz zeigten auch, dass das Kochen während der Fällung, oder das des rasch gefällten Magnesiumammoniumphosphats, höhere Zahlen für das Magnesiumpyrophosphat zur Folge hat.

Der Niederschlag, welcher bei den Bedingungen dieser Versuchsreihe gebildet wird, zeigt im Vergleich mit dem Niederschlag aus der 
ersten Versuchsreihe die folgenden Eigentümlichkeiten: 1. sein Volumen ist grösser ; 2. seine Neigung, an den Glaswänden zu haften, ist gleichfalls grösser; 3. er erglüht gar nicht oder nur teilweise beim Kalzinieren; 4. das aus ihm erhaltene Magnesiumpyrophosphat ist weiss, sein Volumen ist gross und seine Struktur kristallinisch.

Der Niederschlag bei den Versuchen 3 und 4 (Tabelle 12) zeigte deutlich heterogene Struktur. Er war im Anfang lristallinisch, aber es bildete sich beim Kochen ein amorpher Bestandteil, und zuletzt bestand der Niederschlag aus kristallinischen und amorphen Teilen.

\section{4. Übersicht über die Resultate.}

Die Versuche aus dieser Versuchsreibe haben gezeigt, dass unabhängig davon, nach welcher Methode gefällt wird, die analytischen Resultate für die Phosphorsäure von der Temperatur, bei welcher die Fällung ausgeführt wird, abhängig sind. Wie wir gesehen haben, sind die Zahlen für das Magnesiumpyrophosphat höher, wenn während der Fällung die Lösung gekocht wird, oder wenn die Fällungslösung verdünnter ist, und sie sind verbältnismäßjig niedriger, wenn die Menge des Ammoniumchlorids in der Fällungslösung grösser ist.

Es erhebt sich nun die Frage: Welche analytischen Resultate zuverlässiger sind, ob diejenigen, bei welchen die Lösung während der Fällung beständig gekocht wird, oder diejenigen, bei welchen die zu fällende Lösung nur bis zum beginnenden Kochen erwärmt wird. Die äusserst geringe Übereinstimmung der Resultate, welche beim beständigen Kochen der Lösung während der Fällung erhalten werden, ist eine Tatsache, welche allein genügt, um zu zeigen, dass beim Fällen unter solchen Temperaturbedingungen die analytischen Resultate unzuverlässig sind.

Der Umstand, dass die analytischen Resultate zu hoch ausfallen, wenn während der Fällung gekocht wird, zeigt unzweifelhaft, dass dies sekundäre Vorgänge hervorruft. Es fragt sich nun, welcher Natur diese Vorgänge sind. Alle Tatsachen sprechen dafür, dass die sekundären Vorgänge nicht der Ionenzusammensetzung der Lösungen zuzuschreiben sind. Die Versuche 7,8 und 9 aus den Tabellen 10, 11 und 12 zeigen, dass die analytischen Resultate stark davon abhängen, ob der Niederschlag nach der Fällung mit der Flüssigkeit gekocht wird oder nicht. Hiervon ausgehend, sind wir gezwungen, anzunehmen, dass beim Kochen des Niederschlags von Magnesiumammoniumphosphat seine Zu- 
sammensetzung so verändert wird, dass sein Gewicht zunimmt. Die Ursache der Zunahme kann entweder in der Flüssigkeit oder aber in dem Niederschlag selbst gesucht werden.

Um mit der ersten Annahme die in Frage kommende Tatsache zu erklären, müssen wir annebmen, dass das überschüssige Magnesiumchlorid beim Kochen hydrolisiert wird. Um zu sehen, ob diese Annahme zutrifft, habe ich einige von den bei dieser Versuchsreihe erhaltenen Filtraten andauernd und stark gekocht. Es zeigte sich, dass die Lösungen vollständig klar bleiben, oder dass kein Magnesiumhydrat gefällt wird, trotzdem die Flüssigkeiten einen grossen Überschuss an Magnesiumchlorid enthielten. Es bleibt also anzunehmen, dass die Ursache für die Gewichtszunahme in den Eigenschaften des Magnesiumammoniumphosphats zu suchen ist. Durch die hohe Temperatur kann das Magnesiumainmoniumphosphat in folgender Weise hydrolisiert werden:

1. $3 \mathrm{Mg} \mathrm{NH}_{4} \mathrm{PO}_{4}=\mathrm{Mg}_{3}\left(\mathrm{PO}_{4}\right)_{2}+\left(\mathrm{NH}_{4}\right)_{3} \mathrm{PO}_{4}$;

2. $\mathrm{Mg} \mathrm{NH} \mathrm{PO}_{4}+\mathrm{H}_{2} \mathrm{O}=\mathrm{Mg}(\mathrm{OH})_{2}+\mathrm{NH}_{4} \mathrm{H}_{2} \mathrm{PO}_{4}$.

Da diese Vorgänge bei Gegenwart von Magnesiumchlorid, Ammoniumchlorid und Ammoniak verlaufen, ist es verständlich, dass diese eine Zunahme der Niederschlagsmenge zur Folge haben werden.

Wahrscheinlicher aber ist, dass die Zersetzung des Magnesiumammoniumphosphats nach der ersten Gleichung stattfindet. Hierfür sprechen folgende Tatsachen: 1. Das Maguesiumpyrophosphat aus den Versuchen, bei welchen sehr hohe Resultate erhalten wurden, gibt mit einer neutralen Silbernitratlösung eîne deutliche Phosphatreaktion; 2. es ist bekannt, dass das saure Magnesiumphosphat, $\mathrm{MgHPO}_{4}$, beim Kochen mit Wasser in Magnesiumphosphat, $\mathrm{Mg}_{3}\left(\mathrm{PO}_{4}\right)_{2}$, übergeht; 3. es folgt aus den Versuchen von K. B u be (l. c.), dass das Magnesiumammoniumphosphat bei langem Stehen mit Wasser so verändert wird, dass die Menge des Magnesiums im Niederschlag zunimmt.

Aus meinen Versuchen ist zu schliessen, dass die Hydrolyse des Magnesiumammoniumphosphats vollständiger verläuft, a) wenn die Flüssigkeit weniger Ammoniumchlorid enthält und b) wenn die zu fällende Lösung verdünnter ist.

Dritte Versuchsreihe.

\section{Fallungen bei einer Temperatur von $85^{\circ}$.}

Meine in der ersten Versuchsreihe angeführten Bestimmungen zeigten, dass die analytischen Resultate von der Fällungsdauer abhängig sind: 
Beim raschen Fällen nach den Methoden von Järvin en und Jörge n sen sind die Zahlen für das Magnesiumpyrophosphat etwas höher und beim raschen Fällen nach der Methode von Schmitz etwas niedriger als beim langsamen Fällen unter denselben Versuchsbedingungen und nach denselben Methoden. Andererseits zeigten die Versuche der zweiten Versuchsreihe, dass die Temperatur eine wichtige Rolle bei der Bestimmung der Phosphorsäure als Magnesiumpyrophosphat spielt. Es ergibt sich nun die Frage: Ist der Unterschied zwischen den Resultaten der raschen und der langsamen Fällungen nicht auf die verschiedene Temperatur zurückzuführen? Dass die Temperatur, bei welcher die Fällung erfolgt, nicht gleich ist, zeigen folgende Tatsachen. Bei den langsamen Fällungen nimmt die Temperatur der Fällungslösung $a b$ : 1. infolge ihrer Mischung mit dem kalten Fällungsmittel and 2. infolge der Wärmeausstrahlung während des Fällens. Bei den raschen Fällungen nimmt die Temperatur der Fällungslösung nur infolge der niedrigen Temperatur des Fällungsmittels ab. Also ist es klar, dass beim langsamen Fällen der Fällungsvorgang bei höherer Temperatur anfängt und bei viel niedrigerer Temperatur endet als beim raschen Fällen. Überhaupt verläuft der Fällungsvorgang beim raschen Fällen bei höherer Temperatur, als beim langsamen Fällen.

Um zu sehen, ob dieser Unterschied in den Versuchsbedingungen einen Unterschied in den analytischen Resultaten bewirkt, habe ich. andere Versuche angestellt, bei welchen die Fällungslösung nur bis auf $85^{\circ}$ erwärmt und gleich hierauf gefällt wurde. Dadurch verfolgte ich den Zweck, ungefähr solche Versuchsbedingungen zu schaffen, dass die raschen Fällungen bei derselben mittleren Temperatur verlaufen, wie: die "entsprechenden langsamen Fällungen der ersten Versuchsreihe.

Tabelle 13.

Versuche nach der Methode von Järvinen.

\begin{tabular}{|c|c|c|c|c|c|c|c|}
\hline \multirow{2}{*}{ Nr. } & \multicolumn{3}{|c|}{ Fällungslösung } & \multicolumn{3}{|c|}{ Fällungsmittel } & \multirow{2}{*}{$\begin{array}{c}\text { Gefunden } \\
\mathrm{Mg}_{2} \mathrm{P}_{2} \mathrm{O}_{7} \\
g\end{array}$} \\
\hline & $\begin{array}{c}\mathrm{H}_{3} \mathrm{PO}_{4} \\
c c m \\
\end{array}$ & $\begin{array}{r}\mathrm{H}_{2} \mathrm{O} \\
\mathrm{ccm} \\
\end{array}$ & $\begin{array}{r}\mathrm{NH}_{3} \\
\mathrm{ccm}\end{array}$ & $\begin{array}{c}\mathrm{MgCl}_{2} \\
\mathrm{cem} \\
\end{array}$ & $\begin{array}{c}\mathrm{NH}_{4} \mathrm{Cl} \\
\quad \mathrm{ccm} \\
\end{array}$ & $\begin{array}{c}\mathrm{NH}_{3} \\
\mathrm{ccm} \\
\end{array}$ & \\
\hline 1 & 50 & 200 & 5 & 50 & 30 & 15 & 0,4348 \\
\hline 2 & 50 & 100 & 5 & $\breve{50}$ & 100 & 15 & 0,4334 \\
\hline 3 & 50 & 100 & 5 & 100 & 30 & 15 & 0,4363 \\
\hline
\end{tabular}


Versuche nach der Methode von Jörgensen.

\begin{tabular}{|c|c|c|c|c|c|c|}
\hline \multirow{2}{*}{$\mathrm{Nr}$. } & \multicolumn{4}{|c|}{ Fällungslösung } & \multirow{2}{*}{$\begin{array}{c}\text { Fällungs- } \\
\text { mittel } \\
\mathrm{MgCl}_{2} \\
\mathrm{ccm}\end{array}$} & \multirow{2}{*}{$\begin{array}{c}\text { Gefunden } \\
\mathrm{Mg}_{2} \mathrm{P}_{2} \mathrm{O} \\
g\end{array}$} \\
\hline & $\begin{array}{c}\mathrm{H}_{3} \mathrm{PO}_{4} \\
\mathrm{ccm}\end{array}$ & $\begin{array}{r}\mathrm{H}_{2} \mathrm{O} \\
\mathrm{ccm}\end{array}$ & $\begin{array}{l}\mathrm{NH}_{3} \\
\mathrm{ccm}\end{array}$ & $\begin{array}{c}\mathrm{NH}_{4} \mathrm{Cl} \\
\mathrm{ccm}\end{array}$ & & \\
\hline 4 & 50 & 200 & 20 & 30 & 50 & 0,4344 \\
\hline 5 & 50 & 100 & 20 & 100 & 50 & $0,4330^{\circ}$ \\
\hline 6 & 50 & 100 & 20 & 30 & 100 & $0, \pm 357$ \\
\hline
\end{tabular}

Versuche nach der Methode von Schmitz.

\begin{tabular}{|c|c|c|c|c|c|c|c|c|}
\hline \multirow{2}{*}{$\mathrm{Nr}$} & \multicolumn{5}{|c|}{ Fällungslösung } & \multicolumn{2}{|c|}{ Fällungsmittel } & \multirow{2}{*}{$\begin{array}{c}\text { Gefunden } \\
\mathrm{Mg}_{2} \mathrm{P}_{2} \mathrm{O}_{7} \\
g\end{array}$} \\
\hline & $\begin{array}{c}\mathrm{H}_{3} \mathrm{PO}_{4} \\
-\mathrm{ccm} \\
\end{array}$ & $\begin{array}{r}\mathrm{H}_{2} \mathrm{O} \\
\mathrm{ccm}\end{array}$ & $\begin{array}{c}\mathrm{MgCl}_{2} \\
\mathrm{ccm}\end{array}$ & $\begin{array}{c}\mathrm{NH}_{4} \mathrm{Cl} \\
c \operatorname{cs} n\end{array}$ & $\begin{array}{r}\mathrm{HCl} \\
\mathrm{ccm}\end{array}$ & $\begin{array}{c}\text { I. } \mathrm{NH}_{3} \\
\mathrm{ccm}\end{array}$ & $\begin{array}{c}\text { II. } \mathrm{NH}_{3} \\
\mathrm{ccm}\end{array}$ & \\
\hline 7 & 50 & 200 & 50 & 30 & 1 & 50 & 20 & 0,4330 \\
\hline 8 & 50 & 100 & 50 & 100 & 1 & 50 & 20 & $0,43 \supseteq 8$ \\
\hline 9 & 50 & 100 & 100 & 30 & 1 & 50 & 20 & 0,4340 \\
\hline
\end{tabular}

Diese Versuche zeigen, dass, unabhängig von der Fällungsmethode, bei Gegenwart grosser Mengen von Ammoniumchlorid die analytischen Resultate niedriger und bei Gegenwart grosser Mengen von Magnesiumchlorid die Resultate höher sind. Auch in diesem Falle sind die Zahlen für das Magnesiumpyrophosphat beim raschen Fällen nach der Methode von Schmitz niedriger, als beim raschen Fällen nach den Methoden von Järvinen und Jörgensen. Vergleicht man die Resultate dieserVersuche mit denjenigen aus der ersten Versuchsreihe, so kommt man zu dem Schluss, dass, wenn die Fällung nach den Methoden von Järvinen, Jörgensen oder $S \mathrm{chmitz}$ rasch ausgeführt wird, man fast dieselben Zahlen für das Magnesiumpyrophosphat erhält, unabhängigdavon, ob die zu fällende Lösung bis zum beginnenden Sieden oder bloss auf $85^{\circ}$ erwärmt worden ist.

Es folgt also daraus, dass die Differenz zwischen den Resultaten der raschen und der langsamen Fällungen. der ersten Versuchsreihe nicht auf Temperaturunterschiede zurückzuführen ist. Eine andere wahrscheinliche Annahme zur Erklärung der in Frage kommenden Tatsache schien mir die- 
folgende. Da bei den langsamen Fällungen die Niederschläge entweder gleich im Kristallzustand erhalten werden oder schnell in den Kristallzuștand übergehen and bei den raschen Fällungen die Niederschläge erst amorph sind und dann gewöbnlich langsam in den Kristallzustand übergehen, kann angenommen werden, dass dieser Unterschied in den Eigenschaften der Niederschläge die Ursache für die Lifferenzen zwischen den Resultaten der raschen und der langsamen Fällungen ist. Man könnte z. B. annehmen, dass die amorphen Niederschläge, welche beim raschen Fällen ausfallen, von den Stoffen adsorbieren, in deren Gegenwart sie entstehen, und dass sie die adsorbierten Stoffe auch nach ihrem Übergang in den Kristallzustand nicht abgeben. Da die Kontrolle dieser Annahme möglich war, habe ich entsprechende Versuche angestelit.

Wenn die obige Annahme richtig ist, so ist zu erwarten, dass die Differenzen zwischen den Resultaten der raschen und der langsamen Fällungen verschwinden, wenn der Fällungsvorgang so ausgeführt wird, dass der Niederschlag beim raschen Fällen gleich in Kristallzustand erhalten wird. Wie erwähnt, erhält man beim raschen Fällen nach der Methode von Schmitz den Niederschlag gleich im Kristallzustand, wenn die zu fällende Lösung grosse Mengen von Ammoniumchlorid oder Ammoniummolybdat enthält. Auch bei Gegenwart von nicht sehr viel Ammoniummolybdat erhält man beim raschen Fällen nach der Methode von Schmitz den Niederschlag gleich im Kristallzustand, wenn die Fällung unter bestimmten Bedingungen ausgeführt wird.

Diese Tatsachen wurden benutzt, am festzustellen, ob beim raschen Fällen nach der Methode von Schmitz die Zahlen für das Magnesiumpyrophosphat verschieden sein werden, je nachdem der Niederschlag erst amorph erhalten wird und dann in den Kristallzustand übergeht, oder gleich im Kristallzastand erhalten wird. Diese Versuche wurden auch bei einer Temperatur von $85^{0}$ ausgeführt. Ich werde nur ihre Resultate mitteilen. Sie haben gezeigt, dass beim raschen Fällen nach der Methode von Schmitz, unabhängig davon, ob der Niederschlag gleich im Kristallzustand erhalten wird oder nicht, die analytischen Resultate etwas niedriger sind als beim langsamen Fällen nach derselben Methode. Dieselben Versuche zeigten noch: Wenn die Făllung bei Gegenwart von Ammoniummolybdat so ausgeführt wird, dass, bevor das Magnesiumammoniumphosphat entsteht, der Niederschlag von Ammoniumphosphormolybdat vollständig im Ammoniak gelöst wird, erhält man 
nach der Methode von Schmitz etwas niedrigere Zahlen für das Magnesiumpyrophosphat. Wir haben gesehen, dass im umgekehrten Falle nach der Methode von Schmitz höhere analytische Resultate erhalten werden.

\section{Vierte Versuchsreibe.}

\section{Fällung der Phosphorsäure bei gewöhnlicher Temperatur.}

Die Ergebnisse der beschriebenen Versuche lassen noch folgende Fragen ungelöst: 1. Warum sind für die Fällung der Phosphorsäure als Magnesiumammoniumphosphat drei neue Methoden angegeben, während es für die analytische Praxis gleichgültig ist, nach welcher Methode die Fällung ausgeführt wird? 2. Wenn die Fällung unter bestimmten Temperaturbedingungen ausgeführt werden soll, welches ist die günstigste Temperatur? 3. Warum wird vorgeschrieben, die Fällung der Phosphorsäure langsam oder sehr langsam (Methode von Schmitz!) auszuführen, während die Versuche zeigen, dass der Einfluss der Fällungsdauer auf die analytischen Resultate nicht gross ist?

Zur Lösung dieser Fragen habe ich noch Versuche bei gewöhnlicher Temperatur angestellt. Diese Versuche sind auf ähnliche Weise ausgeführt, wie die mitgeteilten. Auch in diesem Falle wurden die Fällungslösung und das Fällungsmittel auf drei verschiedene Weisen bereitet, welche den Vorschriften von Jä̈rinen, Jörgensen und Schmitz entsprechen. Da die Fällangen bei gewöhnlicher Temperatur geschahen, ist es nicht richtig, diese Versuche als nach den Methoden dieser Verfasser ausgeführt zu bezeichnen. Der Kürze wegen werde ich sie aber so bezeichnen.

\section{Tabelle 14.}

Langsame Fällungea bei gewöhnlicher Temperatur. Nach Järvinen.

\begin{tabular}{|c|c|c|c|c|c|c|c|}
\hline \multirow{2}{*}{$\mathrm{Nr}$. } & \multicolumn{3}{|c|}{ Fällungslösung } & \multicolumn{3}{|c|}{ Fällungsmittel } & \multirow{2}{*}{$\begin{array}{c}G e f u n d e n \\
\mathrm{Mg}_{2} \mathrm{P}_{2} \mathrm{O}_{7} \\
g\end{array}$} \\
\hline & $\begin{array}{c}\mathrm{H}_{3} \mathrm{PO}_{4} \\
\mathrm{ccm} \\
\end{array}$ & $\begin{array}{r}\mathrm{H}_{2} \mathrm{O} \\
\mathrm{ccm} \\
\end{array}$ & $\begin{array}{l}\mathrm{NH}_{3} \\
\mathrm{ccm}\end{array}$ & $\begin{array}{c}\mathrm{Mg} \mathrm{Cl}_{2} \\
\mathrm{ccm}\end{array}$ & $\begin{array}{c}\mathrm{NH}_{4} \mathrm{CI} \\
c c m\end{array}$ & $\begin{array}{l}\mathrm{NH}_{3} \\
. \mathrm{ccm}\end{array}$ & \\
\hline 1 & 50 & 200 & 5 & 50 & 30 & 15 & 0,4334 \\
\hline 2 & 50 & 100 & 5 & 50 & 100 & 15 & 0,4301 \\
\hline 3 & 50 & 100 & 5 & 100 & 30 & 15 & 0,4325 \\
\hline 4 & 50 & 100 & 0 & 50 & 30 & 20 & 0,4292 \\
\hline
\end{tabular}

Fresenius, Zeitschrift f. anal. Chemie. LVI. Jahrgang. 11. 11. 12. Heft. $\$ 4$ 
Nach Jörgensen,

\begin{tabular}{|c|c|c|c|c|c|c|}
\hline \multirow{2}{*}{ Nr. } & \multicolumn{4}{|c|}{ Fällungslösung } & \multirow{2}{*}{$\begin{array}{c}\text { Fällungs- } \\
\text { mittel } \\
\mathrm{Mg} \mathrm{Cl}_{2} \\
\mathrm{ccm}\end{array}$} & \multirow{2}{*}{$\begin{array}{c}\text { Gefunden } \\
\mathrm{Mg}_{2} \mathrm{P}_{2} \mathrm{O}_{7} \\
g\end{array}$} \\
\hline & $\begin{array}{c}\mathrm{H}_{3} \mathrm{PO}_{4} \\
-\mathrm{c} m \\
\end{array}$ & $\begin{array}{l}\mathrm{H}_{2} \mathrm{O} \\
c c m\end{array}$ & $\begin{array}{c}\mathrm{NH}_{4} \mathrm{Cl} \\
\mathrm{ccm} \\
\end{array}$ & $\begin{array}{l}\mathrm{NH}_{3} \\
\mathrm{cem}\end{array}$ & & \\
\hline 5 & 50 & 200 & 30 & 2) & 50 & 0,4300 \\
\hline 6 & 50 & 100 & 100 & 20 & 50 & 0,4252 \\
\hline 7 & 50 & 100 & 30 & 20 & 100 & 0,4277 \\
\hline 8 & 50 & 100 & 60 & 20 & 50 & 0,4265 \\
\hline
\end{tabular}

Nach Schmilz.

\begin{tabular}{|c|c|c|c|c|c|c|c|c|}
\hline \multirow{2}{*}{ Nr. } & \multicolumn{5}{|c|}{ Fällungslösung } & \multicolumn{2}{|c|}{ Fällungsmittel } & \multirow{2}{*}{$\begin{array}{c}\text { Gefunden } \\
\mathrm{Mg} \cdot \mathrm{P}_{2} \mathrm{O}_{7} \\
g\end{array}$} \\
\hline & $\begin{array}{c}\mathrm{H}_{3} \mathrm{PO}_{4} \\
\mathrm{ccm}\end{array}$ & $\begin{array}{l}\mathrm{H}_{2} \mathrm{O} \\
\mathrm{ccm} \\
\end{array}$ & $\begin{array}{c}\mathrm{NH}_{4} \mathrm{Cl} \\
\mathrm{ccm}\end{array}$ & $\begin{array}{c}\mathrm{Mg} \mathrm{Cl} \\
\mathrm{ccm}\end{array}$ & $\begin{array}{l}\mathrm{HCl} \\
\mathrm{ccm}\end{array}$ & $\begin{array}{c}\text { I. } \mathrm{NH}_{3} \\
\text { ccm }\end{array}$ & $\begin{array}{c}\text { II. } \mathrm{NH}_{3} \\
\mathrm{ccm}\end{array}$ & \\
\hline 9 & 50 & 200 & 30 & 50 & 1 & 50 & 20 & 0,4338 \\
\hline 10 & 50 & 100 & 100 & 50 & 1 & 50 & 20 & 0,4334 \\
\hline 11 & 50 & 100 & 30 & 100 & 1 & 50 & 20 & 0,4339 \\
\hline
\end{tabular}

Bemerkung. Die Fällungsdauer war bei sämtlichen Versuchen $15 \mathrm{Min}$.

Ein oberflächlicher Blick auf die Resultate dieser Versuche zeigt, dass, wenn die Fällung langsam erst bei gewöhlicher Temperatur ausgeführt wird, die Zahlen für das Magnesiumpyrophosphat sehr verschieden ausfallen. Aus den Versuchen 1 bis 4 ist zu erseben: 1. dass der Wert für das Magnesiumpyrophosphat am grössten ist, wenn die zu fällende Lösung am verdünntesten ist; 2. dass er höher ist, wenn das Fällungsmittel mehr Magnesiumchlorid enthält, und niedriger, wenn das Fällungsmittel mehr Ammoniumchlorid enthält; 3. dass für das Magnesiumpyrophosphat die niedrigste Zahl erhalten wird, wenn die Fällungslösung kein Ammoniak enthält. Durch Vergleichen der Resultate dieser Versuche mit denjenigen der Tabelle 2 kommt man zu dem Schluss, dass wenn die Fällung nach der Methode von Järvinen bei gewöhnlicher Temperatur durchgeführt wird, auch die höchste Zahl für das Magnesiumpyrophosphat niedriger ist, als die Zahlen, welche beim Fällen bei hoher Temperatur erhalten werden. 
Die Versuche (4 bis 8 ) nach Jörgensen zeigen: 1. dass die Zahl für das Magnesiumpyrophosphat am höchsten ist, wenn die Fällungslösung am stärksten verdünnt ist; 2. dass grosse Mengen von Ammoniumchlorid niedrigere Zahlen für das Magnesiumpyrophosphat zur Folge haben und 3. dass, wenn die Menge des Magnesiumchlorids grösser ist, die Zahl für das Magnesiumpyrophosphat höher ist. Ein Vergleich der Resultate dieser Versuche mit denjenigen aus Tabelle 5 zeigt, dass beim langsamen Fällen nach der Methodevon Jörgensen für das Magnesiumpyrophosphat niedrigere Zahlen erhalten werden, wenn die Fällung bei gewöhlicher Temperatur ausgeführtwird, als wenn unter sonst gleichen Bedingungen die Fällung bei hoher Temperatur ausgeführt wird. Aus Tabelle 14 ist noch zu ersehen, dass, wenn bei gewöhnlicher Temperatur langsam gefällt wird, man für das Magnesiumpyrophosphat niedrigere Zahlen erhält beim Fällen nach der Methodevon Jörgensen als beim Fällen nach Järvinen. Zuletzt sieht man aus den Versuchen nach Schmitz (9 bis 11), dass die Zahlen für das Magnesiumpyrophosphat nichl so stark von der Menge der sichan dem Fällungsvorgang beteiligenden Stoffen abhängig sind. Mit den entsprechenden Versuchen aus Tabelle 8 verglichen, zeigen diese Versuche, dass wenn die Fällung langsam nach der Methode von Schmitz ausgeführt wird, für das Magnesiumpyrophosphat etwas höhere Zahlen erhalten werden, wenn bei hoher Temperatur gefällt wird.

Der Niederschlag, welcher entsteht, wenn bei gewöhnlicherTemperatur nach Järvinen and $J$ örgen sen gefällt wird, ist sehr feinkristallinisch. Er gibt beim Waschen trübe Filtrate. Um klare Filtrate zu erhalten, muss wiederholt filtriert and sehr vorsichtig ausgewaschen werden. Das nach Schmitz erhaltene Magnesiumammoniumphosphat ist ebenso feinkristallinisch, aber die Filtrate sind klar.

Um festzustellen, ob die niedrigeren Resultate, welche beim Fällen nach Järvinen und Jörgensen erhalten werden, nicht der unvollständigen Fällung der Phosphorsäure zuzuschreiben sind, habe ich die Filtrate von einigen Versuchen verdampft, die Ammoniumsalze verjagt, den Rückstand in Salpetersäure gelöst, filtriert und mit Ammoniummolybdat auf Phosphorsäure geprüft. Es wurde nur eine schwache gelbe Färbung erhalten, eine Trübung der Lösung aber wurde auch 
nach 24 Stunden nicht beobachtet. Dieselbe Erscheinung zeigt sich, wenn Filtrate von Versuchen, bei welchen heiss gefällt wurde, auf ähnliche Weise geprüft werden. Es folgt daraus, dass die Ursache der niedrigeren Resultate beim Fällen bei gewöhnlicher Temperatur nicht der unvollständigen Fällung der Phosphorsäure zuzuschreiben ist.

Tabelle 15.

Rasche Fällungen bei gewöhnlicher Temperatur. Nach Järvinen.

\begin{tabular}{|c|c|c|c|c|c|c|c|}
\hline \multirow{2}{*}{ Nr. } & \multicolumn{3}{|c|}{ Fällungslösung } & \multicolumn{3}{|c|}{ Fällungsmittel } & \multirow{2}{*}{$\begin{array}{c}\text { Gefunden } \\
\mathrm{Mg}_{2} \mathrm{P}_{2} \mathrm{O}_{7} \\
-\quad g \\
\end{array}$} \\
\hline & $\begin{array}{c}\mathrm{H}_{3} \mathrm{PO}_{4} \\
\mathrm{ccm} \\
\end{array}$ & $\begin{array}{r}\mathrm{H}_{2} \mathrm{O} \\
\mathrm{ccm}\end{array}$ & $\begin{array}{r}\mathrm{NH}_{3} \\
\mathrm{ccm}\end{array}$ & $\begin{array}{c}\mathrm{MgCl}_{2} \\
\mathrm{ccm}\end{array}$ & $\begin{array}{c}\mathrm{I}^{2} \mathrm{H}_{4} \mathrm{Cl} \\
:{ }^{\prime} n\end{array}$ & $\begin{array}{r}\mathrm{NH}_{3} \\
\mathrm{ccm}\end{array}$ & \\
\hline 1 & 50 & 100 & 5 & 50 & 30 & 15 & 0,4342 \\
\hline 2 & 50 & 200 & 5 & 50 & 30 & 15 & 0,4346 \\
\hline 3 & 50 & 300 & 5 & 50 & 30 & 15 & $0 ; 4339$ \\
\hline 4 & 50 & 100 & 5 & 50 & 100 & 15 & 0,4336 \\
\hline 5 & 50 & 100 & 5 & 100 & 30 & 15 & 0,4347 \\
\hline
\end{tabular}

$\mathrm{Nach} \mathrm{Jörgensen.}$

\begin{tabular}{|c|c|c|c|c|c|c|}
\hline \multirow{2}{*}{ Nr. } & \multicolumn{4}{|c|}{ Fällungslösung } & \multirow{2}{*}{$\begin{array}{c}\text { Fällungs- } \\
\text { mittel } \\
\mathrm{Mg} \mathrm{Cl}_{2} \\
\mathrm{ccm}\end{array}$} & \multirow{2}{*}{$\begin{array}{c}\text { Gefunden } \\
\mathrm{Mg}_{2} \mathrm{P}_{2} \mathrm{O}_{7} \\
g\end{array}$} \\
\hline & $\begin{array}{c}\mathrm{H}_{3} \mathrm{PO}_{4} \\
c c m\end{array}$ & $\begin{array}{r}\mathrm{H}_{\mathrm{s}} \mathrm{O} \\
\mathrm{cem} \\
\end{array}$ & $\begin{array}{c}\mathrm{NH}_{4} \mathrm{Cl} \\
\mathrm{ccm} \\
\end{array}$ & $\begin{array}{l}\mathrm{NH}_{3} \\
\mathrm{ccm}\end{array}$ & & \\
\hline 6 & 50 & 100 & 30 & 20 & 50 & 0,4344 \\
\hline 7 & 50 & 200 & 30 & 20 & 50 & 0,4344 \\
\hline 8 & 50 & 300 & 30 & 20 & 50 & 0,4340 \\
\hline 9 & 50 & 100 & 100 & 20 & 50 & 0,4334 \\
\hline 10 & 50 & 100 & 30 & 20 & 100 & 0,4354 \\
\hline
\end{tabular}

Nach Schmitz.

\begin{tabular}{|c|c|c|c|c|c|c|c|c|}
\hline \multirow[b]{2}{*}{ Nr. } & \multicolumn{5}{|c|}{ Fällungslösung } & \multicolumn{2}{|c|}{ Fällungsmittel } & \multirow{2}{*}{$\begin{array}{c}\text { Gefunden } \\
\mathrm{Mg} \mathrm{P}_{2} \mathrm{O}_{7} \\
g\end{array}$} \\
\hline & $\begin{array}{c}\mathrm{H}_{3} \mathrm{PO}_{4} \\
\mathrm{ccm}\end{array}$ & $\begin{array}{r}\mathrm{H}_{2} \mathrm{O} \\
\mathrm{ccm}\end{array}$ & $\begin{array}{c}\mathrm{NH}_{4} \mathrm{Cl} \\
\mathrm{ccm}\end{array}$ & $\begin{array}{c}\overline{\mathrm{Mg} \mathrm{Cl}} \\
\mathrm{ccm}\end{array}$ & $\begin{array}{r}\mathrm{HCl} \\
\mathrm{ccm}\end{array}$ & $\begin{array}{l}\text { I. } \mathrm{NH}_{3} \\
\text { ccm }\end{array}$ & $\begin{array}{c}\text { II. } \mathrm{NH}_{\mathbf{3}} \\
\mathrm{ccm}\end{array}$ & \\
\hline 11 & 50 & 200 & 30 & 50 & 1 & 50 & 20 & 0,4343 \\
\hline 12 & 50 & 300 & 30 & 50 & 1 & 50 & 20 & 0,4337 \\
\hline 13 & 50 & 100 & 100 & 50 & 1 & 50 & 20 & 0,4330 \\
\hline 14 & 50 & 100 & 30 & 100 & 1 & 50 & 20 & 0,4350 \\
\hline
\end{tabular}


Man sieht aus dieser Tabelle, dass, wenn die Phosphorsäure bei gewöhnlicher Temperatur, aber rasch gefällt wird, für das Magnesiumpyrophosphat bei sonst gleichen Versuchsbedingungen fast dieselben Zahlen erhalten werden, unabhängig davon, ob die Fällung nach Järvinen, Jörgensen oder Schmitz ausgeführt worden ist. Diese Versuche zeigen noch, dass die Zahlen für das Magnesiumpyrophosphat niedriger sind, wenn die Fällungslösung stärker verdünnt ist, oder wenn die Menge des Ammoniumchlorids sehr gross ist, und höher sind, wenn die Menge des Maguesiumchlorids grösser ist.

Ein Vergleich der Versuche aus den Tabellen 14 und 15 zeigt: 1. dass, wenn die Phosphorsäure bei gewöhnlicher Temperatur gefällt wird, die Fällungsdauer eine wichtige analytische Rolle spielt; 2. während

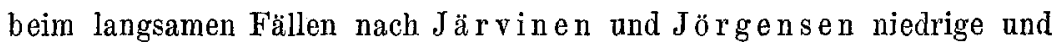
nicht übereinstimmende Resultate erhalten werden, stimmen beim raschen Fällen die Resultate gut überein; 3. beim raschen Fällen nach Sch mitz sind die Zahlen für das Magnesiumpyrophosphat etwas höher als beim langsamen Fällen.

Der wesentliche Unterschied zwischen den Resultaten aus Tabelle 15 und denjenigen aus den Tabellen $3,6,9,2,5$ und 8 besteht darin, dass die Zahlen für das Magnesiumpyrophosphat aus Tabelle 15 untereinander besser übereinstimmen. Mit anderen Worten gesagt, wenn die Phosphorsäure als Magnesiumammoniumphosphat bei gewöhnlicher Temperatur rasch gefällt wird, so sind die analytischen Resultate nicht so stark abhängig von der Menge des Magnesiumchlorids und des Ammoniumchlorids, wie das der Fall ist, wenn die Fällung bei hoher Temperatur ausgeführt wird.

Die Niederschläge, welche beim raschen Fällen bei gewöhnlicher Temperatur erhalten werden, sind sehr feinkörnig, sie geben aber trotzdem keine trüben Filtrate. Die nach Järvinen und Jörgensen erhaltenen Niederschläge zeigen die folgende besondere Eigenschaft. Wenn man sie, nachdem sie im Trockenschrank getrocknet sind, auf ein mit Ledertuch gereinigtes Chrglas legt, springen einem kleine Teilchen des Niederschlages entgegen, sobald das Uhrglas aufgehoben wird. Diese für die analytische Praxis unangenehme Eigenschaft des Niederschlags wird beseitigt, wenn das Uhrglas vorher mit direkter Flamme etwas erwarmt wird. Aus dieser Tatsache ist zu schliessen; dass die Erscheinung elektrischer Natur ist. 


\section{E. Zusammenfassung und allgemeine Übersicht über die Resultate.}

Die systematischen Untersuchungen über die Methoden zur Bestimmling der Phosphorsäure als Magnesiumpyrophosphat ergaben folgende Resultate :

1. Wenn die zu fällende Lösung bis zum beginnenden Sieden erhitzt wird und, ohne weiter zu erwärmen, gefällt wird, so erhält man beim langsamen Fällen nach den Methoden von Järvinen, Jörgensen und Schmitz annähernd gleiche analytische Resultate. Diese Resultate schwanken je nach der quantitativen Zusammensetzung der Lösungen in verschiedenen Richtungen. Der Unterschied zwischen den Resultaten liegt fast in den Grenzen der Vorsuchsfehler, wenn die zu fällende Lösung mit verschiedenen Mengen Wasser verdünnt ist.

Die Resultate sind höher: a) wenn die zu fällende Lösung Kaliumchlorid enthält; b) wenn die Menge des Magnesiumchlorids gross ist; c) wenn die Menge des Ammoniaks gross ist und d) wenn beim Fällen nach der Methode von Schmitz die Fällungslösung Ammoniummolybdat enthält.

Die Resultate sind niedriger: a) wenn die Menge des Ammoniumchlorids gross ist und b) wenn beim Fällen nach der Methode von Järvinen und Jörgensen die zu fällende lösung grosse Mengen Ammoniummolybdat enthält.

Die Gegenwart von Natriumchlorid in der zu fällenden Lösung übt keinen Einfluss auf die analytischen Resultate aus.

Beim raschen Fällen der Phosphorsäure unter denselben Temperaturbedingungen erhält man nach den Methoden von $J a ̈ r v i n e n$ und Jörgensen übereinstimmende und nach der Methode von Schmitz niedrigere Resultate. Bei den raschen Fällungen nach den Methoden von $J$ ärvinen und Jörgensen sind die analytischen Resultate etwas höher als diejenigen, welche bei den langsamen Fällungen nach derselben Methode erhalten werden. Bei den raschen Fällungen nach der Methode von Schmitz sind die Resultate etwas niedriger, als diejenigen, welche bei den langsamen Fällungen nach demselben Verfahren erhalten werden.

Die Verdünnung der Fállungslösung, die Menge des Magnesiumchlorids, Ammoniumchlorids, Ammoniummolybdats, Ammoniaks und ebenso die Gegenwart von Kaliumchlorid und Natriumchlorid in der zu fällenden Lôsung beeinflusst die analytischen Resultate bei den raschen Fällungen nach den Methoden von Järvinen, Jörgensen und Schmitz auf dieselbe Weise, wie bei den langsamen Fällungen nach denselben Methoden. 
2. Wenn während der Fällung der Phosphorsäure die Fällungslösung beständig im Sieden gehalten wird, oder wenn der Niederschlag von Magnesiumammoniumphosphat mit der Flüssigkeit, aus der er entstanden ist, gekocht wird, erhält man nach den Methoden von Järvinen, Jörgensen und Schmitz höhere analytische Resultate, als wenn während der Fällung nicht gekocht wird. Die unter solchen Temperaturbedingungen erhaltenen Resultate sind böher, wenn die Fällungslösung verdünnter ist, und sie sind verhältnismäfig niedriger, wenn die zu fällende Lösung grössere Mengen Ammoniumchlorid enthält.

3. Wenn die Fällung der Phosphorsäure aus einer Lösung, welche bis $85^{\circ}$ erwärmt ist, geschieht, so erhält man beim raschen Fällen nach den Methoden von Järvinen, Jörgensen und Schmitz annähernd solche analytischen Resultate, wie sie beim raschen Fällen bis zum beginnenden Sieden der Fällungslösung nach denselben Methoden erzielt werden.

Rasche Fällungen nach der Methode von Schmitz (bei $85^{\circ}$ ), welche unter solchen Bedingungen ausgeführt sind, dass das Magnesiumammoniumphosphat direkt im Kristallzustand erbalten wurde, haben gezeigt, dass die Resultate dieser Methode nicht davon abhängig sind, ob der Niederschlag erst amorph erhalten wird und dann in den Kristallzustand übergeht, oder direkt im Kristallzustand ausfällt.

4. Wenn der Fällungsvorgang bei gewöhnlicher Temperatur ausgeführt wird, erhält man beim langsamen Fällen nach Järvinen und Jörgensen sebr niedrige und nicht übereinstimmende Resultate und beim langsamen Fällen nach $\mathrm{Schmitz}$ verhältnismäßig höhere und besser übereinstimmende Resultate. Auch in diesem Falle bewirkt eine grosse Menge von Ammoniumchlorid niedrigere and grosse Menge von Magnesiumchlorid höhere Zahlen für das Magnesiumpyropbosphat.

Wird die Fällung der Phosphorsäure wieder bei gewöhnlicher Temperatur, aber rasch ausgeführt, so sind die Resultate unter sonst gleichen Bedingungen unabhängig davon, nach welcher Methode gefällt wird. Diese Versuche zeigten noch, dass eine starke Verdünnung der zu fällenden Lösung oder grosse Mengen von Ammoniumchlorid niedrigere Zablen und eine grosse Menge von Magnesiumchlorid höhere Zahlen für das Magnesiumpyrophosphat zur Folge haben. Der Einfluss dieser 
Faktoren auf die analytișchen Resultate ist aber verhältnismäßig gering, infolgedessen erhält man für die Phosphorsäure die am besten übereinstimmenden Resultate, wenn die Fällung bei gewöhnlicher Temperatur rasch ausgeführt wird.

Diese Untersuchungen führten $z \mathfrak{u}$ folgenden Schlüssen betreffs des Fällungsvorgangs :

1. Wird die Fällung der Phosphorsäure aus einer Lösung, welche bis zum beginnenden Sieden erwärmt ist, ausgeführt, so verlaufen keine sekundären Fällungsvorgänge, unabhängig davon, nach welcher Methode gefällt wird. Die Tatsache, dass die analytischen Resultate schwankend sind, wenn die Lösungen verschiedene Mengen von Magnesiumchlorid, Ammoniumchlorid, Ammoniak und Ammoniummolybdat enthalten, kann befriedigend mit der Löslichkeit des unter diesen Bedingungen entstandenen Magnesiumammoniumchlorids erklärt werden.

2. Zur Erklärung des Befundes, dass die Gegenwart von Kaliumchlorid in der Fällungslösung höhere analytische Resultate bedingt, haben wir angenommen, dass unter diesen Bedingungen sekundäre Fällungsvorgänge verlaufen, deren Produkt ein Kalium enthaltendes Magnesiumphosphat ist.

3. Wird während der Fällung das Erhitzen der Lösung nicht unterbrochen, oder wird der Niederschlag von Magnesiumammoniumphosphat mit der Flüssigkeit, aus der er entstanden ist, gekocht, so wird das Magnesiumammoniumphosphat teilweise nach folgender Gleichung zersetzt:

$$
3 \mathrm{Mg} \mathrm{NH} \mathrm{PO}_{4}=\mathrm{Mg} 3\left(\mathrm{PO}_{4}\right)_{2}+\left(\mathrm{NH}_{4}\right)_{3} \mathrm{PO}_{4} .
$$

Es bleibt uns noch übrig, festzustellen, zu welchen Schlüssen bezüglich des Fällungsvorganges die Versuche bei gewöhnlicher Temperatur führen.

Die Taksache, dass, wenn die Fällung langsam nach Järvinen ausgeführt wird, die analytischen Resultate so stark von der Menge der an dem Fällungsvorgang beteiligten Verbindungen abhängig sind, ist nur mit der Annahme erklärlich, dass beim Fällen unter solchen Bedingungen sekundäre Fällungsvorgänge verlaufen. Da hierbei die analytischen Resultate überhaupt niedrig sind, ist anzunehmen, dass das sekundäré Produkt $\mathrm{Mg}\left[\left(\mathrm{NH}_{4}\right)_{2} \mathrm{PO}_{4}\right]_{2}$ und der sekundäre Fällungsvorgang

$$
2\left(\mathrm{NH}_{4}\right)_{2} \mathrm{PO}_{4}{ }^{\prime}+\mathrm{Mg} \ddot{*}=\mathrm{Mg}\left[\left(\mathrm{NH}_{4}\right)_{2} \mathrm{PO}_{4}\right]_{2}
$$

sein wird (siehe S. 499). 
Mit dieser Annahme sind die Resultate der Versuche nach Järvinen aus Tabelle 14 leicht erklärlich. Z. B. beim Versuch 1 ist die Fällung aus verhältnismäßig stark verdünnter Lösung ausgeführt; folglich kann die Konzentration der Ionen $\left(\mathrm{NH}_{4}\right)_{2} \mathrm{PO}_{4}^{\prime}$ nicht gross sein, und der sekundäre Vorgang, welcher durch diese Ionen hervorgerufen wird, wird. in unbedentendem Maße verlaufen. Beim Versuch 2 ist die zu fällende Lösung konzentrierter und die Menge des Ammoniumchlorids im Fällungsmittel grösser. Es ist klar, dass die beiden Bedingungen eine grössere Konzentration der Anionen $\left(\mathrm{NH}_{4}\right)_{2} \mathrm{PO}_{4}{ }^{\prime}$ zur Folge haben werden; infolgedessen muss bei diesem Versuch ein niedrigerer Wert für das Magnesiumpyrophosphat erwartet werden. Der Versuch 3 unterscheidet sich von Versuch 2 durch die Menge des Ammoniumchlorids im Fällungsmittel. Da die Menge des Ammoniumchlorids bei diesem Versuch kleiner ist, muss ein grösserer Wert für das Magnesiumpyrophosphat erwartet werden als bei Versuch $2^{1}$ ). Der Versuch 4 ist ausgeführt, um den Einfluss zu erforschen, welchen die Gegenwart von Ammoniak in der zu fällenden Lösung ausübt. Die Zahl für das Magnesiumpyrophosphat ist bei diesem Versuch die niedrigste. Die Ursache dieser Tatsache wird klar, wenn man berücksichtigt, dass die Phosphorsäure ein schwächerer Elektrolyt ist, als ihre $\gamma$-Salze.

Alle diese theoretischen Folgerungen wurden durch die Versuchsergebnisse bestätigt. Neue Beweise für die Wahrscheinlichkeit der Annahme, dass beim langsamen Fällen der Phosphorsäure bei gewöhnlicher Temperatur das sekundäre Produkt $\mathrm{Mg}\left[\left(\mathrm{NH}_{4}\right)_{2} \mathrm{PO}_{4}\right]$ entsteht,

1) Es scheint, dass für das höhere Resultat des Versuchs 3 (Tab. 14) auch die Menge des Magnesiumchlorids im Fällungsmittel von Belang ist. Für diese Annahme spricht die folgende Tatsache. Wie erwähnt, trübt sich das Filtrat beim Waschen der Niederschläge aus diesen Versuchen. Diese Trübung wird nicht durch die Feinkörnigkeit des Niederschlages hervorgerufen, da, wenn die Fällung bei gewöhnlicher Temperatur rasch ausgeführt wird, die Niederschläge feinkörniger sind, und doch werden die Filtrate nicht trüb. Diese Tatsache führt zu dem Schluss, dass die Trübung bei den Versuchen aus Tabelle 14 der Löslichkeit des sekundären Produkts $\mathrm{Mg}\left[\left(\mathrm{NH}_{4}\right)_{2} \mathrm{PO}_{4}\right]_{2}$ zuzuschreiben ist. Angenommen, dass diese Verbindung in hohem Maßse löslich ist, wird es klar, dass bei Gegenwart eines Überschusses des Fällangsmittels das sekundäre Produkt in Magnesiumammoniumphosphat äbergehen wird und dass dieser Vorgang vollständiger verlaufen wird, wenn die Lösung grössere Mengen von Magnesiumchlorid enthält. Wir werden sehen, dass auch andere Tatsachen zu dem Schlass führen, dass das sekundäre Produkt $\mathrm{Mg}\left[\left(\mathrm{NH}_{4}\right)_{2} \mathrm{PO}_{4}\right]_{2}$ in hohem Maße löslich ist. 
bieten die Versuche nach Jörgensen aus Tabelle 14. Theoretisch sind die Bedingungen für die Entstehung dieses sekundären Produkts günstiger, wenn die Fällung uach der Methode von Jörgensen ausgeführt wird. Infolgedessen müssen die analytischen Resultate niedriger sein, wenn unter sonst gleichen Bedingungen die Fällung nicht nach Järvinen, sondern nach Jörgensen ausgeführt wird. Diese theoretische Folgerung wird durch die Versuchsergebnisse völlig bestätigt.

Es bleibt uns noch übrig, die Resultate zu erklären, welche beim langsamen Fällen nach Schmitz erhalten werden (Tabelle 14). Wie wir im allgemeinen Teil gesehen haben, bewirkeı die besonderen Bedingungen, unter welchen die Fällung nach $\mathrm{Schmitz}$ geschieht, einen anderen Verlauf der chemischen Umsetzungen. Wenn bei der theoretischen Betrachtung dieser Vorgänge die Löslichkeit der denkbaren sekundären Produkte berücksichtigt' wird, kommt man zum Schluss, dass beim Fällen nach Schmitz manche sekundären Vorgänge leseitigt oder begrenzt werden müssen. Das wird durch die Tatsachen bestätigt. Es scheint aber, dass die verhältnismäßig höheren und besser äbereinstimmenden Resultate, welche beim langsamen Fällen nach Schmitz bei gewöhnlicher Temperatur erhalten werden, nicht nur der Löslichkeit der theoretisch denkbaren sekundären Produkte, sondern auch dem Umstand zuzuschreiben sind, dass beim Fällen nach Schmitz der Fällungsvorgang immer bei Gegenwart eines grossen Magnesiumchloridüberschusses verläuft. Denn unter solchen Bedingungen ist die Möglichkeit zur Entstehung derartiger sekundärer Produkte, welche weniger Magnesium enthalten, als der Formel $\mathrm{Mg} \mathrm{NH} \mathrm{PO}_{4}$ entspricht, viel geringer. Für diese Annahme spricht auch die Tatsache, dass, wenn die Fällung nach $J a ̈ r v i n e n$, aber bei Abwesenheit von Ammoniak in der zu fällenden Lösung ausgeführt wird, die analytischen Resultate viel niedriger sind (Versuch 4, Tabelle 14), als wenn die Fällung nach Schmitz ausgeführt wird.

. Es entsteht nun die Frage: Wenn die Erklärung der Tatsachen aus Tabelle 14 richtig ist, warum ist dieselbe Erklärung nicht auch für den Fall anwendbar, wenn die Fällung nach den Methoden von Järvinen, Jörgensen und Schmitz langsam, aber bei hoher Temperatur ausgeführt wird? Die Ursache dieser Tatsache kann nur in dem Einfluss, welchen die hohe Temperatur auf die Löslichkeit des sekundären Produktes $\mathrm{Mg}\left[\left(\mathrm{NH}_{4}\right)_{2} \mathrm{PO}_{4}\right]_{2}$ ausübt, gesucht werden. Die Tatsachen zwingen zu der Annahme, dass die Löslichkeit dieses Produktes 
mit der Temperatur zunimmt, und infolgedessen wird durch die hohe Temperatur der Übergang desselben in Magnesiumammoniumphosphat erleichtert. Diese Tatsachen führen also zu dem Schluss, dass durch Erhöhung der Temperatur manche sekundären Vorgänge beseitigt werden können, für deren Eintritt die gewöhnliche Temperatur günstig ist.

Grössere Schwierigkeiten bietet die Erklärung der Vorgänge bei den Versuchen der Tabelle 15. Es folgt aus diesen Versuchen, dass, wenn die Fällung der Phosphorsäure bei gewöhnlicher Temperatur, aber rasch ausgeführt wird, die Zahlen für das Magnesiumpyrophosphat höher sind, als wenn unter denselben Bedingungen langsam gefällt wird. Dieser Befund widerspricht der Theorie.

Einen ähnlichen Widerspruch zwischen der Theorie und den Tatsachen habe ich bei meinen Untersuchungen über die Bestimmung des Baryums als Baryumsulfat gefunden ${ }^{1}$ ). Zur Erklärung desselben habe ich angenommen, dass der Kristallzustand der Niederschläge dabei eine Rolle spielt. Beim langsamen Fällen von Baryumchlorid mit Schwefelsäure erhält man den Niederschlag in grosskörnigerem Zustand, infolgedessen kann der Überschuss des Fällungsmittels nicht auf das sekundäre Fällungsprodukt wirken. Beim raschen Fällen sind die Niederschläge feinkörniger, ihre Oberfläche ist also grösser, und das begünstigt die Überführung des sekundären Produktes in das Hauptprodukt.

Die Analogie zwischen dieser Tatsache und derjenigen, die sich bei der Fällung der Phosphórsäure zeigt, ist vollkommen. Bei der Fällung der Phosphorsäure werden die sekundären Vorgänge in derselben Weise durch die Ionenzusammensetzung der zu fällenden Lösung hervorgerufen wie beim Fällen des Baryums; auch beim raschen Fällen der Phosphorsäure sind die Niederschläge feinkörniger als beim langsamen Fällen; schliesslich sind die analytischen Resultate bei der Bestimmung der Phosphorsäure beim raschen Fällen ebenfalls höher. Von dieser Analogie ausgehend, kommt man zu dem Schluss, dass beim raschen Fällen der Phosphorsäure bei gewöhnlicher Temperatur die analytischen Resultate deswegen höher sind, weil infolge der grösseren Niederschlagsoberfläche dem Überschuss des Fällungsmittels die Möglichkeit gegeben wird, das entstandene sekundäre Fällungsprodulst in das Hauptprodukt überzuführen.

1) Diese Ztschrft. 57, 77 und 113 (1917). 
Eine andere wahrscheinliche Erklärung derselben Tatsache ist die folgende. Aus der Zusammensetzung des sekundären Produktes. $\mathrm{Mg}\left[\left(\mathrm{NH}_{4}\right)_{2} \mathrm{PO}_{4}\right]_{2}$ ist $\mathrm{zu}$ ersehen, dass eine Bedingung $\mathrm{zu}$ seiner Entstehung eine unzureichende Menge von Magnesium ist. Diese Bedingung ist am besten erfüllt beim langsamen Fällen. Beim raschen Fällen erfolgt der Fällungsvorgang bei Gegenwart eines grossen Magnesiumchloridüberschusses, und demzufolge sind die Bedingungen für die Entstehung des sekundären Produktes $\mathrm{Mg}\left[\left(\mathrm{NH}_{4}\right)_{2} \mathrm{PO}_{4}\right)_{2}$ ungünstig. Die Tatsachen, über die ich verfüge, reichen aber nicht aus für die Entscheidung der Frage, welche von diesen Annahmen wahrscheinlicher ist.

Die verhältnismäßig grössere Úbereinstimmung der analytischen Resultate, welche beim raschen Fällen bei gewöhlicher Temperatur erhalten werden, ist ein Zeichen, dass unter diesen Versuchsbedingungen der Fällungsvorgang am regelmäßigsten verläuft. Hierfür sprechen noch folgende Tatsachen. Wir haben gesehen, dass, wenn während der Fällung der Phosphorsäure die Lösung gekocht wird, die Zahlen für das Magnesiumpyrophosphat ungewöhnlich hoch sind. Als Ursache für diese Tatsache haben wir die Zersetzung des Magnesiumammoniumphosphats angesehen. Es fragt sich nun: Erfolgt solche Zersetzung auch dann nicht, wenn die zu fällende Lösung nur bis zum beginnenden Sieden erwärmt und dann gefällt wird? Dass solche Zersetzung auch bei solchen Versuchsbedingungen, wenn auch in geringerem Maßse, stattinden wird, beweisen die Tatsachen, dass die Resultate dieser. Versuche etwas höher sind, wenn die $\mathrm{zu}$ fällende Lösung verdünnter ist (Tabellen 1 und 3) und wenn die Menge des Magnesiumchlorids gross ist (Tabellen 2, 5, 8).

Es folgt aus dieser Übersicht, dass die Ergebnisse meiner Versuche über die Bestimmung der Phosphorsäure als Magnesiumpyrophosphat ohne die Annahme, dass Magnesiumbydrat als sekundäres Produkt gefällt wird, befriedigend erklärlich sind. Meine Versuchsergebnisse sprechen auch gegen die Behauptung K. Bubes (1. c.), dass beim Fällen von Phosphorsäure als Magnesiumammoniumphosphat gleichzeitig Ammoniumphosphat, $\left(\mathrm{NH}_{4}\right)_{3} \mathrm{PO}_{4}$, gefällt wird, und bringen neue Beweise für die. Entstehung des sekundären Produktes $\mathrm{Mg}\left[\left(\mathrm{NH}_{4}\right)_{2} \mathrm{PO}_{4}\right]_{2}$.

Zuletzt wollen wir noch die in praktischer Beziehung wichtige Frage betrachten: Bei welchen Versuchsbedingungen die Phosphorsäure als Magnesiumammoniumphosphat gefällt werden muss, um die zuverlässigsten analytischen Resultate zu ergeben. Trotzdem aus meinen 
Versuchen mit grosser Wahrscheinlichkeit geschlossen werden kann, dass der Fällungsvorgang am regelmäbigsten verläuft, wenn die Fällung bei gewöhnlicher Temperatur rasch ausgeführt wird, genügen sie doch nicht zur endgültigen Lösung dieser Frage.

Wir haben gesehen, dass die analytischen Resultate bei der Bestimmung der Phosphorsäure als Magnesiumpyrophosphat abhängig sind. 1. von der Temperatur, bei welcher gefällt wird; 2. von der Fällungsdauer; 3. von der Menge des angewandten Magnesiumchlorids, Ammoniumchlorids usw. und 4. von der Fällungsmethode. Es ist wahr, dass die Differenzen zwischen den analytischen Resultaten in den meisten Fällen sehr klein sind, jedoch kann nicht sicher entschieden werden, welche Resultate dem wahren Gehalt an Phosphorsäure entsprechen. Zur Entscheidung dieser Frage kann eine andere Methode als Kontrollmethode nicht benutzt werden, da eine genauere Methode zur Bestimmung der Phosphorsäure nicht bekannt ist. Andererseits ist die Auswahl einer solchen Grundsubstanz, deren Phosphorsäuregehalt genan bekannt ist, schwierig. Neue Untersuchungen, welche die Lösung dieser Frage bezwecken, sind im Gange.

Sofia, Chemisches Institut der Universität.

\section{Berioht über die Fortschritte der analytischen Chemie.}

I. Allgemeine analytische Methoden, analytische 0perationen, Apparate und Reagenzien.

1. Auf theoretische und physikalische Chemiebezügliche. Von

R. Fresenius und L. Fresenius.

Gasanalyse, Wasserstoff und Kohlenwasserstoffe. Da das bei der Wasserstoffbestimmung nach Paal and Hartmann ${ }^{1}$ ) als Katalysator zur Wasserstoffübertragung verwendete Palladiumsol recht teuer geworden ist, haben E. Bosshard und E. Fis chli ${ }^{2}$ ) nach einem anderen ähnlichen Verfahren gesucht und ein solches in Anlehnung an das Verfahren der Fetthärtung ${ }^{3}$ ) gefunden. Als leicht reduzierbare Substanz bewährte sich am besten Natriumoleat in konzentrierter wässriger Lösung, der $3 \%$ Nickel als Wasserstoffüberträger zugesetzt waren. Die Bereitung dieses Katalysators muss mit besonderer Sorgfalt geschehen. Die Ver-

1) Vergl. diese Ztschrft. 54, 505 (1915). - 2) Ztschrft. f. angew. Chem. 28, 365 (1915). — 3) Vergl. diese Ztschrft. 55, 65 (1916). 